\title{
Hybrid Integration of Magnetoresistive Sensors with MEMS as a Strategy to Detect Ultra-Low Magnetic Fields
}

\author{
João Valadeiro ${ }^{1,2, *}$, Susana Cardoso ${ }^{1,2}$, Rita Macedo ${ }^{3}$, Andre Guedes ${ }^{3}$, João Gaspar ${ }^{4}$ and \\ Paulo P. Freitas 1,4 \\ 1 Instituto de Engenharia de Sistemas de Computadores-Microsystems and Nanotechnology (INESC-MN), \\ Rua Alves Redol, No. 9, Lisboa 1000-029, Portugal; scardoso@inesc-mn.pt (S.C.); pfreitas@inesc-mn.pt (P.P.F.) \\ 2 Instituto Superior Técnico IST, Physics Department, Universidade de Lisboa, Lisbon 1049-001, Portugal \\ 3 Picosense, Inc., 1900 Addison St Ste 200, Berkeley, CA 94704, USA; ritamacedo@gmail.com (R.M.); \\ andre.aires@gmail.com (A.G.) \\ 4 International Iberian Nanotechnology Laboratory (INL), Av. Mestre Jose Veiga, Braga 4715-330, Portugal; \\ Joao.Gaspar@inl.int \\ * Correspondence: jvaladeiro@inesc-mn.pt; Tel.: +351-21-310-0348; Fax: +351-21-314-5843
}

Academic Editor: Stephanus Büttgenbach

Received: 21 March 2016; Accepted: 4 May 2016; Published: 11 May 2016

\begin{abstract}
In this paper, we describe how magnetoresistive sensors can be integrated with microelectromechanical systems (MEMS) devices enabling the mechanical modulation of DC or low frequency external magnetic fields to high frequencies using MEMS structures incorporating magnetic flux guides. In such a hybrid architecture, lower detectivities are expected when compared with those obtained for individual sensors. This particularity results from the change of sensor's operating point to frequencies above the $1 / f$ noise knee.
\end{abstract}

Keywords: magnetoresistive sensors; noise; MEMS; hybrid devices; frequency modulation; magnetic field detection

\section{Introduction}

The detection of low-intensity magnetic fields (down to pico-Tesla range) plays a major role in pushing the limits and widen the use of magnetic sensors in industrial applications $[1,2]$ from automotive control to non-destructive tests (NDT) [3], being pivotal in the biomedical field, where it stands out in the bio-molecular recognition [4] and bio-signals imaging [5]. Both magnetic field sensitivity and intrinsic noise level set the sensor's minimum field detected, being the latter the focus of this review. Presently, magnetic field sensing technologies include: superconducting quantum interference devices (SQUIDs) — the uppermost technique used for neuroimaging; fluxgate magnetometers [6] — prevalent in military industry (e.g., aircraft compass heading systems); Hall effect sensors [7]—widely use in low cost position sensor applications and Magnetoresistive (MR) sensors [8] - traditionally integrated in data storage industry [9].

The ability to operate at room temperature combined with (i) a high signal to noise ratio (SNR) in a large bandwidth (from DC to hundreds of $\mathrm{MHz}$ ); (ii) a small footprint ( $\mu \mathrm{m}$ scale lithographed areas) and (iii) low power consumption $(\sim \mathrm{mW})$ allowed MR sensors to suit a wide range of applications beyond data storage, being a reliable alternative for detection at pico to mili-Tesla range. Detection limits below $\mathrm{pT} / \mathrm{Hz}^{1 / 2}$ have already been demonstrated using MR sensors based on $\mathrm{MgO}$ magnetic tunnel junctions (MTJs) [10]. However, in the low frequency range the intrinsic noise of MR sensors is dominated by the $1 / f$ magnetic component, which limits the minimum detectable field; at high frequency the sensor noise is reduced to the thermal level. 
The use of a large array of small sensing elements connected in series [11] or patterning single sensing elements with larger active areas [12] are among the different strategies used to reduce the noise of MR sensors. On the other hand, instead of acting in the sensor intrinsic noise, a reliable alternative consists in the inclusion of AC microelectromechanical systems (MEMS) resonators with incorporated magnetic flux concentrators (MFCs) to modulate (quasi-)DC signals to an operating frequency (above the $1 / f$ knee) where the thermal-mechanical noise dominates, and the total noise is minimum.

This review focuses on the hybrid integration of MR sensors with MEMS in an attempt to enhance the detection level of the device, exploiting the operation details and advantages of AC modulation at high frequency. This strategy allows magnetic detection of low-intensity and low-frequency signals when compared with conventional techniques centered on the reduction of the sensors intrinsic noise at low frequency.

\section{Background}

\subsection{Magnetoresistive Sensors}

The resistance of a MR sensor changes with the variation of the external magnetic field, being the magnetoresistance value defined as the total resistance variation $(\Delta R)$ normalized to its minimum value $\left(R_{\text {min }}\right)$. Ferromagnetic/non-magnetic heterostructures such as spin-valves (SV) and magnetic tunnel junctions (MTJ) offer advantages over the single ferromagnetic films exhibiting (anisotropic magnetoresistance (AMR)), due to the magnetic stability and larger output signals. Either types of sensing devices are composed by a four layer structure: two ferromagnets (FM) separated by a non-magnetic layer (conductor in SVs; insulator in MTJs) and an antiferromagnet (AFM) responsible for setting the magnetization of a FM layer in a fixed direction (reference) (Figure 1a). In a SV sensor [13] the current flows in plane (CIP) and the resistance variation arises from spin dependent scattering of conduction electrons. In a MTJ, the electrons have to tunnel across the insulating barrier $(<1 \mathrm{~nm}$ thick) as a result of a spin dependent tunneling probability, which originates an electron flow perpendicular to the plane (CPP). While the former display a magnetoresistance around $5 \%-10 \%$-typical structure: $\mathrm{Ni}_{80} \mathrm{Fe}_{20} / \mathrm{Co}_{80} \mathrm{Fe}_{20} / \mathrm{Cu} / \mathrm{Co}_{80} \mathrm{Fe}_{20} / \mathrm{Mn}_{76} \mathrm{Ir}_{24}$ (Figure 1b), the latter are characterized by a larger resistance variation, reaching tunnel magnetoresistance values at room temperature of 50\%-70\% in structures with amorphous AlOx barriers (incoherent electron tunneling) [14] or over 200\% upon annealing with crystalline $\mathrm{MgO}$ barriers (coherent electron tunneling)-typical structure: $\mathrm{Ir}_{20} \mathrm{Mn}_{80} / \mathrm{Co}_{70} \mathrm{Fe}_{30} / \mathrm{Ru} / \mathrm{Co}_{40} \mathrm{Fe}_{40} \mathrm{~B}_{20} / \mathrm{MgO} / \mathrm{Co}_{40} \mathrm{Fe}_{40} \mathrm{~B}_{20} / \mathrm{Ta} / \mathrm{Ni}_{80} \mathrm{Fe}_{20}$ [15]. The signal level achieved is proportional to the magnetoresistance ratio [16], therefore crystalline MTJ devices are pointed as the most promising candidates for a high sensitive field sensor. The sensitivity of a MgO-based MTJ is typically tens of $\% / \mathrm{mT}$, while for a SV sensor is $\sim 1 \% / \mathrm{mT}$. However, extra noise sources, materials and annealing treatments required for a linear response $[17,18]$ are additional issues to be considered in MTJs, when compared to AMR or SV devices. A fair comparison should take into consideration noise levels, to better address the minimum detectable fields [19]. As the intrinsic noise of MTJs is higher than in SV, the former only has a better performance due to higher SNR.

\subsubsection{Noise Sources in MR Sensors}

To measure a magnetic signal of interest, its amplitude must be substantially higher than the devices' noise level. The noise of a MR sensor comes mainly from magnetic fluctuations associated to magnetic domain nucleation and displacement within the sensing layer. The main noise components in a MR sensor are: thermal (electronic) and shot noise contributions (being the latter only present in devices with tunneling mechanism), thermal magnetic noise, random telegraph noise (RTN) and $1 / f$ (electronic and magnetic) noise [20-22]. 


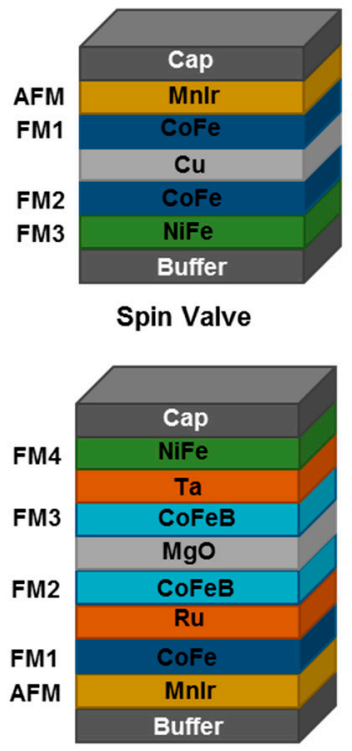

(a) Magnetic Tunnel Junction

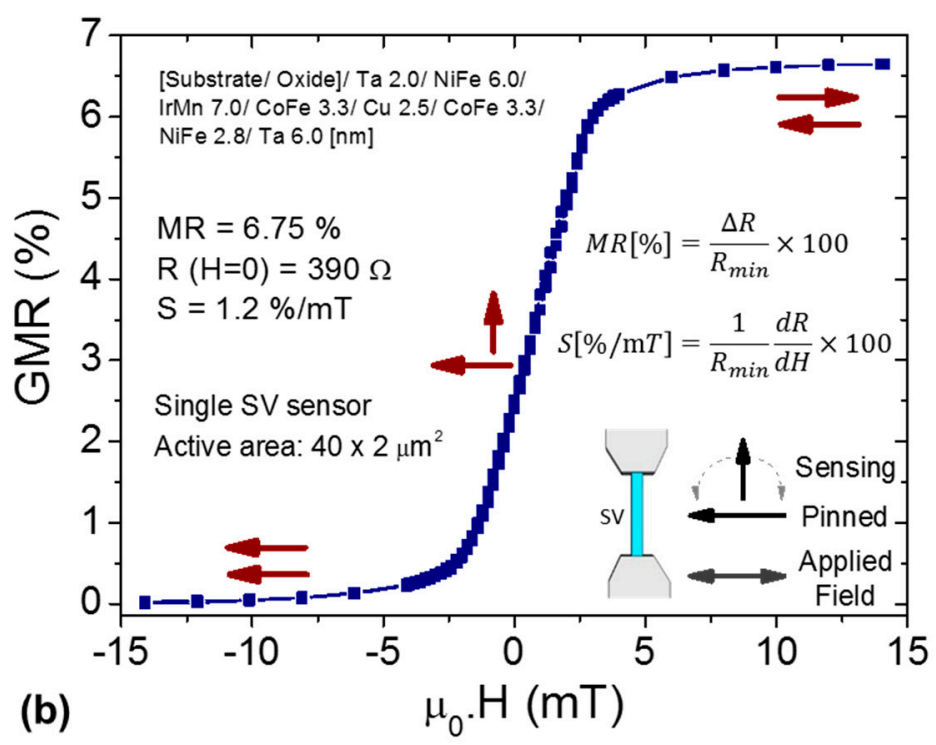

Figure 1. (a) Schematic view of a typical multilayer for spin-valves (SV) and magnetic tunnel junction (MTJ) sensors. SV: antiferromagnet (AFM), pinned layer (FM1), conductive spacer (Cu), sensing layer (FM2 and FM3). MTJ: antiferromagnet (AFM), pinned layer (FM1), insulator spacer (MgO), sensing layer (FM3 and FM4); a synthetic antiferromagnet (SAF) is obtained with the tri-layer FM1/Ru/FM2. The pinned layer magnetization is set in a fixed direction due to exchange-bias at AFM/FM interface; (b) Representative magnetotransport curve obtained for a patterned SV sensor (active area: $40 \times 2 \mu \mathrm{m}^{2}$ ) exhibiting a linear, centered and hysteresis free response. Red arrows illustrate the relative orientation of the in-plane magnetization in both pinned and sensing layers. $S$ stands for the sensor sensitivity.

Thermal (electronic) noise [23] arises from the random thermal motion of electrons responsible for collisions with impurities and other electrons, presenting therefore a dependence on the device resistance $(R)$ and absolute temperature $(T)$, described by the Nyquist formulation:

$$
S_{V}^{t h}\left[\mathrm{~V}^{2} / \mathrm{Hz}\right]=4 k_{B} T R
$$

where $k_{B}$ stands for the Boltzmann constant. This component only vanishes at absolute zero, and shows a flat behavior along the entire frequency band. Once the thermal velocity of electrons is higher than its velocity in the conducting media, $S_{V}^{t h}$ has no dependence on the bias current. Shot noise [24] is caused by circuit discontinuities, in this case the insulating layer in MTJs resulting from the charge carrier's discrete nature, originating a pulsed current and thus exhibiting fluctuations in a short time scale. This fluctuation power density increases with the device bias current (I) and is independent of the frequency:

$$
S_{V}^{s h}\left[\mathrm{~V}^{2} / \mathrm{Hz}\right]=2 q I R^{2}
$$

where $q$ is the electron charge. Shot noise is not present in SV sensor devices.

As the dimensions of a MR sensor are decreased, random fluctuations of the sensing layer magnetization occur under thermal excitation, becoming comparable with the thermal (electronic) noise. These thermally activated fluctuations are the source of thermal magnetic noise, which is frequency independent and inversely proportional to the sensing layer volume $[20,25,26]$.

The random magnetization fluctuations in the sensing layer caused either by a repeated capture of electrons into trapping centers [27] or by the displacement of domain walls [20,28] are the origin of RTN (or Barkhausen effect), which exhibits a Lorentzian type frequency behavior. RTN in not always evident, being shadowed in the low-frequency band by the $1 / f$ magnetic noise. 
The $1 / f$ noise, composed of an electronic and magnetic component, has a spectral density inversely proportional to the frequency, being the dominant source in the low-frequency regime [20,27]. The electronic part ( $1 / f$ electronic) arises from voltage fluctuations related to charge trapping in crystal defects. The oscillations in the sensing layer magnetization caused by domain wall pinning and depinning at defect sites is behind the origin of the magnetic part ( $1 / f$ magnetic) [22]. Therefore, the maximum density of $1 / f$ magnetic noise occurs in the linear transition of the sensor, where the magnetization of the sensing layer is switching between the two saturation states. The $1 / f$ magnetic noise is dominant in the sensor linear range and is mostly suppressed in the saturation states [29]. The $1 / f$ spectral density is empirically described by the Hooge formulation [30]:

$$
\begin{gathered}
S_{V}^{1 / f(S V)}\left[\mathrm{V}^{2} / \mathrm{Hz}\right]=\frac{\gamma_{H}}{N_{c}} \frac{V^{2}}{f} \\
S_{V}^{1 / f(M T J)}\left[\mathrm{V}^{2} / \mathrm{Hz}\right]=\frac{\alpha_{H}}{A} \frac{V^{2}}{f}
\end{gathered}
$$

where $\gamma_{H}\left(\alpha_{H}\right)$ is the (modified) Hooge constant, $N_{c}$ is the number of charge carriers (proportional to pillar area $A$ in a MTJ), $f$ the operating frequency and $V$ the bias voltage. In standard $\mathrm{MgO}-\mathrm{MTJ} \alpha_{H}$ typical values are in the $10^{-9}-10^{-7} \mu \mathrm{m}^{2}$ range $[17,31]$. For MgO-MTJ structures with a soft pinned sensing layer this parameter increases, targeting the $10^{-7}-10^{-5} \mu \mathrm{m}^{2}$ range indicating higher intrinsic noise [17,32-34]. Above the $1 / f$ knee, thermal and shot noise become predominant, overlapping the $1 / f$ spectral density.

The magnetic noise of a MR sensor (comprising both $1 / f$ and thermal magnetic components) shows a linear dependence on the sensitivity [21,26]:

$$
S_{V}^{m a g}\left[\mathrm{~V}^{2} / \mathrm{Hz}\right]=\beta \frac{k_{B} T V^{2}}{\pi \mu_{0} M_{s} \Omega f} \frac{\Delta R}{R}\left(\frac{1}{R} \frac{d R}{d H}\right)
$$

where $\Omega$ and $M_{S}$ are respectively the volume and the saturation magnetization of the sensing layer, $\mu_{0}$ the magnetic permeability of free space and $\beta$ is the ratio between the imaginary and real parts of the sensing layer transverse magnetic susceptibility. Equation (5) is deduced using the fluctuation dissipation relation considering the system in thermal equilibrium [21].

At high-frequency only thermal noise source is present for both SVs and MTJs. Notice that this level is now 2-3 orders of magnitude lower than in low-frequency regime.

Figure 2 shows a representative curve for the noise of a MR sensor (DC to $100 \mathrm{kHz}$ ), where the transition from $1 / f$ domain to the thermal level is clearly observed. One can thus, identify the optimum operation regime with a minimum noise above the $1 / f$ transition. This is not compatible with the detection of low frequency fields, such as for biometric signals or space applications.

\subsubsection{Detection Level Enhancement of MR Sensors}

The minimum detectable field at a specific frequency depends on the sensor's intrinsic magnetic noise (targeted lowest possible) and its sensitivity $(d R / d H)$. On the one hand, particular noise reduction strategies act mainly at the low-frequency range leaving the high-frequency noise almost unchanged. On the other hand, an improvement of the sensor sensitivity also results in an overall detection level enhancement.

For applications that do not require a high spatial resolution, the use of a large array of $\mathrm{N}$ elements connected in series is a reliable alternative to improve the detection limit, since the SNR improves with $\mathrm{N}^{1 / 2}$, although the noise level increases by a factor $\mathrm{N}[11,35]$. A major disadvantage of this approach is the increase in total device resistance, which not only implies a higher thermal noise background but also leads to a more complex integration with electronics. To overcome this problem, the use of a parallel of elements in series instead of a simple series yields a resistance reduction [36]. In addition, patterning a single sensing element with a large area (higher number of carriers) contributes directly 
for the noise level reduction at low frequency $[10,12,18]$. However, large patterned ferromagnetic materials display magnetic domains, hence introducing RTN in the magnetic response.

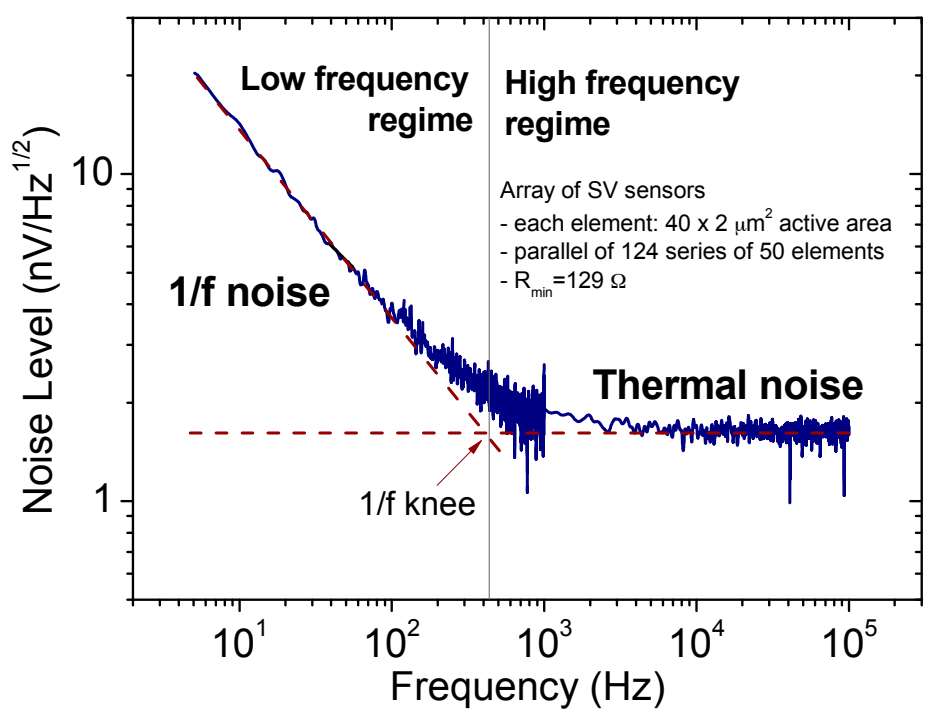

Figure 2. Typical noise spectrum of a MR device (SV array), reaching the thermal level for frequencies around $400 \mathrm{~Hz}$. In the low frequency regime the $1 / f$ noise is dominant, while in the high frequency regime the spectrum is reduced to its thermal level. Both electronic and magnetic noise components are present since the spectrum was recorded with the sensor operating on its linear range.

The integration of MFCs is the used strategy to enhance significantly the sensor sensitivity. These elements increase the magnetic flux through the sensor and consequently decrease the linear operating range without introducing additional noise $[10,12,31,37,38]$. Depending on the geometry and profile, MFCs can yield a sensitivity gain up to 100 times [39], reflecting in a detection level decrease. A brief overview on MFCs integration is presented in Section 2.3.

Table 1 shows typical values of detection level obtained for a single SV sensor (active area: $40 \times 2 \mu \mathrm{m}^{2}$ ) at $30 \mathrm{~Hz}$, where $1 / f$ is dominant; and at $10 \mathrm{kHz}$, in the thermal regime. These are compared with sensor in the gap of funnel shaped MFCs (gain $\sim 10$, gap $=4 \mu \mathrm{m}$ ) and an array of $992 \mathrm{SV}$ elements connected in parallel of series. A bias current of $1 \mathrm{~mA}$ was used in each device.

Table 1. Comparison of typical detection levels of a single SV element, obtained in low $(10 \mathrm{~Hz})$ and high $(10 \mathrm{kHz})$ frequency range, with the obtained results upon the inclusion of MFCs and the patterning of a large array of series connected in parallel of series.

\begin{tabular}{|c|c|c|c|c|c|c|}
\hline Sensor Type & $\begin{array}{c}\text { Individual } \\
\text { Sensor } \\
\text { Area }\left(\mu \mathbf{m}^{2}\right)\end{array}$ & $\begin{array}{c}\text { Device } \\
\text { Footprint } \\
\left(\mu \mathbf{m}^{2}\right)\end{array}$ & $\begin{array}{c}\text { Noise Level } \\
\text { at } 30 \mathrm{~Hz} \\
\left(\mathrm{nV} / \mathrm{Hz}^{1 / 2}\right)\end{array}$ & $\begin{array}{c}\text { Noise Level } \\
\text { at } 10 \mathrm{kHz} \\
\left(\mathrm{nV} / \mathrm{Hz}^{1 / 2}\right)\end{array}$ & $\begin{array}{l}\text { Detectivity } \\
\text { at } 30 \mathrm{~Hz} \\
\left(\mathrm{nT}^{2} \mathrm{~Hz}^{1 / 2}\right)\end{array}$ & $\begin{array}{l}\text { Detectivity } \\
\text { at } 10 \mathrm{kHz} \\
\left(\mathrm{nT} / \mathrm{Hz}^{1 / 2}\right)\end{array}$ \\
\hline Single SV sensor & $40 \times 2$ & $40 \times 2$ & 84.7 & 4.1 & 21.2 & 1.32 \\
\hline Single SV sensor with MFCs & $40 \times 2$ & $400 \times 293$ & 84.7 & 4.1 & 1.9 & 0.12 \\
\hline Array of 992 SV elements & $40 \times 2$ & $886 \times 895$ & 2.4 & 1.3 & 2.2 & 0.61 \\
\hline
\end{tabular}

Increasing the bias current improves the detection level in the high frequency range [32], remaining unchanged below the $1 / f$ knee. Therefore, the use of MR sensors with large current biasing can be faced as a viable alternative for applications requiring an improved detectivity at high frequencies.

Table 1 clearly shows that the detection in the low-frequency regime is limited. A sophisticated way to overcome this low-frequency restriction consists in the integration of MEMS with MFCs and MR sensors, whose motion modulates the interest signal and displaces it to the thermal regime [40,41]. This hybrid strategy is discussed in next sections. 


\subsection{MEMS Resonators}

Microelectromechanical systems (MEMS) resonators have a wide range of applications in transducing technologies [42], either sensing devices with great demand on pressure sensors $[43,44]$ and accelerometers/gyroscopes [45,46], or micro-actuators, taking advantage of a high frequency operation and low power consumption. More recently, MEMS resonating technology has been integrated in more mature sensing applications, as magnetometers based on Lorentzian-force-generated mechanical resonance [47,48], replacing quartz crystal oscillators for signal process [49], or biosensors for label free differentiation of bio-molecules [50-52].

The most common structures used as MEMS resonators are: (i) suspended cantilevers and (ii) torsional bridges, which vibrate at high frequencies ( $\mathrm{MHz}$ range) when actuated by a gate voltage. MEMS torsional paddle bridges-torsional vibrating mode-present higher deflection amplitudes than cantilevers-flexural vibrating mode. The maximum oscillation amplitude corresponding to the most sensitive operation point, occurs at its resonance frequency, and is expressed by:

$$
f_{0}[\mathrm{~Hz}]=\frac{1}{2 \pi} \sqrt{\frac{K}{m}}
$$

where $m$ is total resonator mass and $K$ corresponds to the spring constant (dependent on the resonator dimensions and mechanical properties). A common technique to evaluate the MEMS deflection is measuring the electrical capacitance between the resonator and the gate electrode. In addition, MEMS can behave as actuators since a voltage (both DC and AC) between the resonator and the gate generates an electrostatic force between these two structures (capacitor plates), inducing an oscillation in the former.

The combination of robustness and high sensitivity of MR sensors with the low power consumption and high frequency operation of MEMS resonators results in high frequency magnetic field modulation, deflection detection of micro-bridges [53], and $1 / f$ noise suppression in SVs and MTJs. Microelectromechanical flux concentrator, where MFCs are defined on MEMS flaps can then be integrated with MR sensors. The novelty of this device relies on the mechanical modulation of (quasi-)DC fields at the sensor position achieved by the MFCs oscillation at a frequency corresponding to the MEMS resonance, allowing a shift in the operating point to the high-frequency range $[40,41,54-60]$. A schematic is shown in Figure 3.

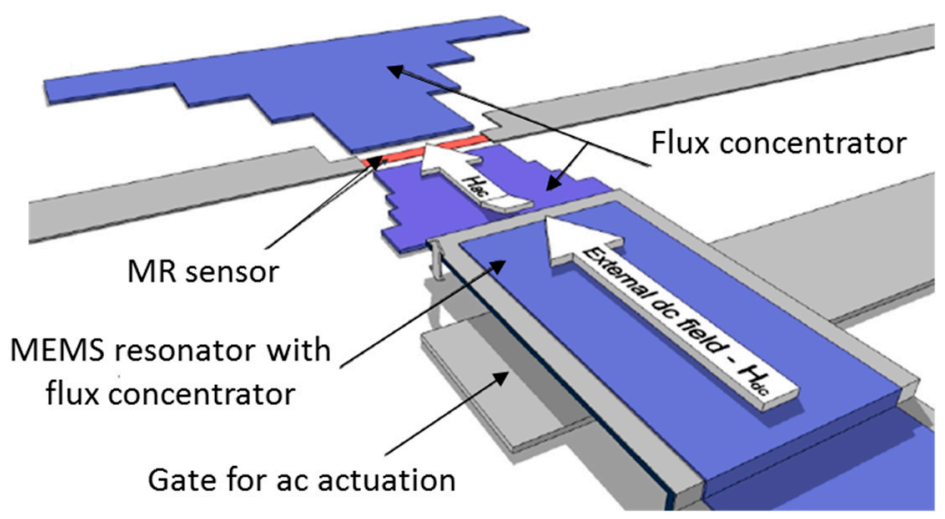

Figure 3. Schematic of a MR-MEMS hybrid device used for high frequency modulation of (quasi-)DC magnetic fields, where a MEMS cantilever with incorporated MFCs oscillates at high frequency to modulate the signal of interest. (Courtesy of Guedes A.).

A different application of a MR-MEMS hybrid device was developed at INESC-MN [61]. In this device, a CoCrPt permanent magnet was defined on top of a cantilever while a SV element was fabricated near the cantilever's free end. Such device showed to be capable of detecting the cantilever's 
mechanical deflection. The field sensed by the SV changes according to the permanent magnet position induced by the deflection. This hybrid device displayed a resolution of $0.06 \AA / \mathrm{Hz}^{1 / 2}$.

An alternative to the described mechanical field modulation consists in the use of chopping techniques to modulate the sensitivity of MR sensors and consequently overcome the $1 / f$ noise [62]. A parallel chopping approach demonstrated a slight reduction of noise spectrum at very low frequency values.

\subsection{Magnetic Flux Concentrators}

The integration of magnetic flux concentrators (MFCs) is a reliable strategy to enhance significantly sensor's sensitivity when the device footprint is not an issue for the application. Being made of soft ferromagnetic materials (e.g., NiFe [63] or amorphous Co based alloys [64]) and with an appropriate geometry, MFCs concentrate the external field in the region where the sensor is placed. The effective gain introduced by the MFCs is defined by the ratio between the magnetic field in the gap and external field. The gain depends on the geometrical parameters (e.g., length, pole distance, yoke/pole ratio-Figure 4a) $[10,17,32,65]$ and intrinsic magnetic properties of the material (e.g., magnetic permeability). Thereby, upon MFCs geometry optimization and material control, a maximum enhancement of the field detection can be achieved. Figure $4 \mathrm{~b}$ illustrates the integration of MFCs with a SV sensor, with focus on the concentrators' gap region where the sensing element is placed.

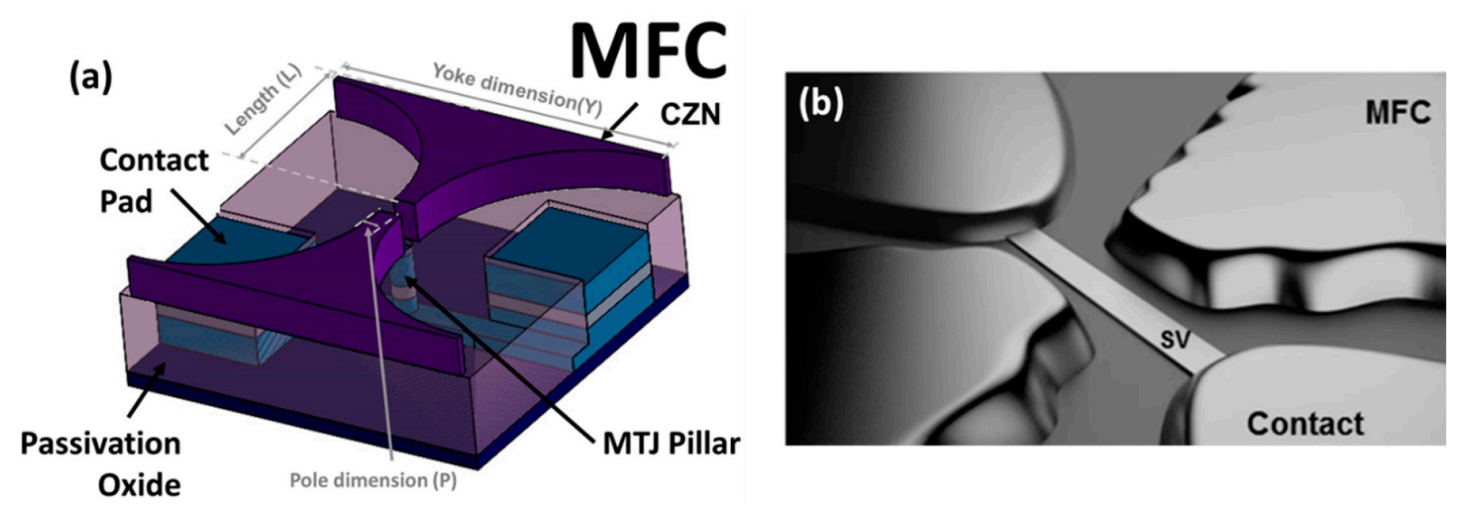

Figure 4. (a) Schematic view of MFCs with funnel shape geometry and respective integration with a patterned MTJ sensor (before the top contact deposition). Reprinted with kind permission of The European Physical Journal (EPJ); (b) Illustration of steep profile MFCs close to a SV single sensor. Reprinted with permission from Leitao D.C. et al., Sensors 2015, 15, 30311-30318. Copyright 2015 MDPI.

The profile of the MFCs also influences the gain of the device. MFCs patterned with a funnel shape and a steep profile $\left(90^{\circ}\right.$, defined by lift-off) in the gap region provided a sensitivity enhancement up to 30 times [12,17,32]. MFCs made of the same magnetic material and with the same pole-sensor distance, but with a $3 \mathrm{D}$ tapered profile at the pole region $\left(45^{\circ}\right.$, defined by ion milling etch), lead to an increased magnetic flux concentration resulting in sensitivity gains up to 100 times [40].

However, an important characteristic of this single layer MFC-MR sensor integration approach, common to both steep and 3D profiles, is a significant thickness difference between MFCs and sensor. This results in major losses of the concentrated flux since it is not captured by the sensing device. To overcome this particularity and guide a larger amount of magnetic flux to the sensor, a more complex structure combining steep and tapered profiles was developed. This new approach is shown in Figure 5a and consists in a double layer MFCs: starting with a thin layer of $\mathrm{Ni}_{80} \mathrm{Fe}_{20}(0.1 \mu \mathrm{m}$ thick, profile angle $\left.45^{\circ}\right)$ in the pole region; and a second layer of $\mathrm{CoZrNb}(0.5 \mu \mathrm{m}$ thick, steep profile). The $\mathrm{CoZrNb}$ pole is separated by $50-100 \mu \mathrm{m}$ from the $\mathrm{Ni}_{80} \mathrm{Fe}_{20}$ pole, reducing considerably the MFCs thickness at the pole region. With this configuration, the field kept by the entire structure is driven into 
the thin $\mathrm{Ni}_{80} \mathrm{Fe}_{20}$ layer, minimizing the flux concentration losses in the sensing region, when compared with traditional single layer MFCs (Figure 5b).

a)
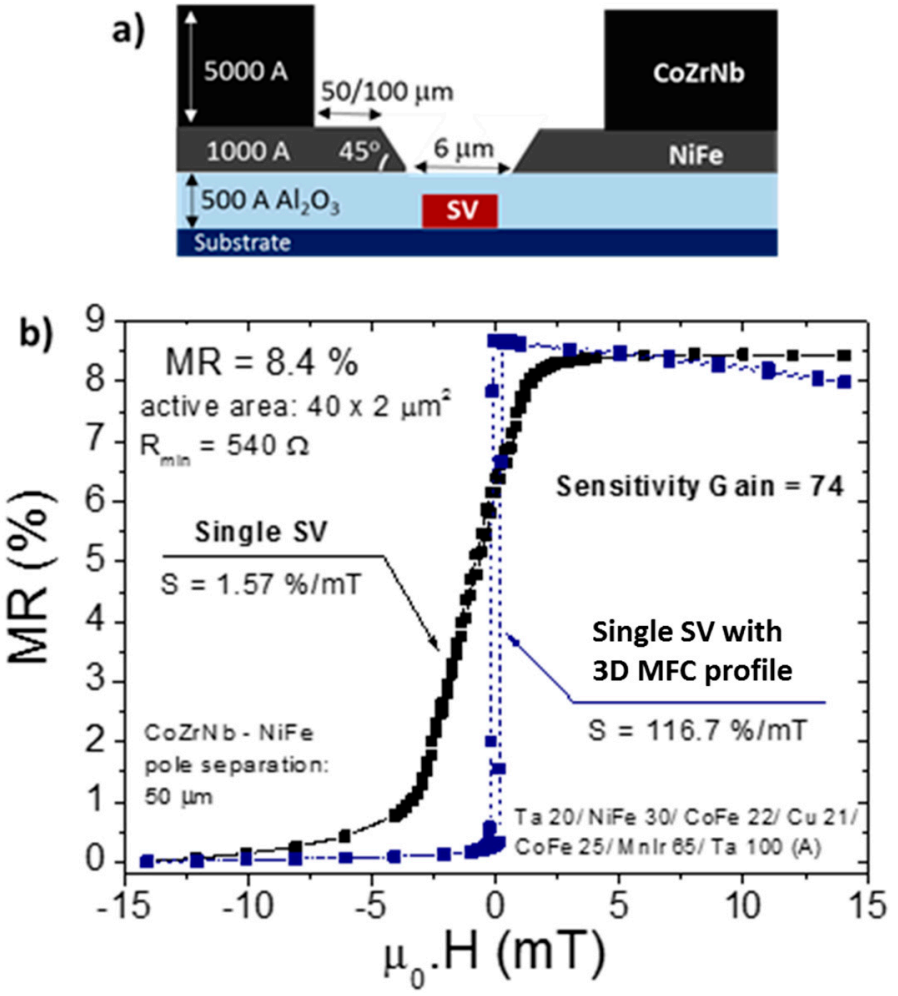

Figure 5. (a) Schematic cross section of the double layer MFC with a 3D tapered profile, showing a pole angle of $45^{\circ}$ in the $\mathrm{NiFe}$ film and a steep profile in the $\mathrm{CoZrNb}$ pole, integrated with a SV sensor; (b) Effect of the 3D tapered profile MFC integration on the magnetotransport curve of a single SV sensor, showing a sensitivity enhancement of 74 times.

\subsection{Hybrid Technology Integrating MR Sensors, MEMS and MFCs}

The MEMS flux concentrator integrated with MR sensors is a reliable solution to overcome the problem imposed by the $1 / \mathrm{f}$ noise level in the detection of magnetic signals in low-frequency regime. Edelstein et al. proposed a design [40,41] consisting of a SV sensor defined between two MFCs $\left(2 \times\left[\mathrm{Cr} 40 \AA / \mathrm{Ni}_{80} \mathrm{Fe}_{20} 1500 \AA\right]\right)$ with trapezoidal shape and deposited on silicon MEMS flaps (MFCs gap $=52 \mu \mathrm{m}$ ), presenting normal-mode resonant frequencies in the $10 \mathrm{kHz}$ range. The oscillatory movement of the MEMS flaps is induced by electrostatic comb drives or by applying an AC voltage between the flap and a gate electrode. This modulates the field in the sensing area shifting the sensor operation point to high frequencies. Recently, a distinct MEMS drive method using piezoelectric materials was introduced [56,58-60]. The output voltage of the MR sensor induced by the resonator with incorporated MFCs has a magnetic contribution arising from the modulated field. For capacitive MEMS, an electric contribution also appears caused by a coupling between the sensor and the resonator gate electrode $[55,57]$. While piezoelectric MEMS oscillates at the same frequency of the driving signal $(f)$, the components of the driving signal influence the motion of capacitive MEMS. For the latter, a pure AC voltage at frequency $f$ causes a vibration at twice the frequency (2f) [53], while an AC voltage with a DC component originates a motion at $f(f$ and $2 f)$ if the voltage offset is higher (lower) than the alternating voltage amplitude.

Depending on the spatial arrangement (e.g., in-plane, over the sensor), the field can be modulated at the oscillating frequency or at twice this value. Considering that the MEMS flaps are in the plane of the sensing element, two cycles of magnetic field (minimum field/maximum field/minimum field) 
occur in each cantilever mechanical cycle (out-of-plane up/in-plane/out-of-plane down). Therefore, when the MEMS flaps are driven to oscillate at their resonance frequency $\left(f_{0}\right)$, a static magnetic field is modulated at $2 f_{0}$-output frequency. However, when a quasi-DC magnetic field (frequency $f_{m}$ ) is the signal of interest, the field modulated at $2 f_{0}$ is in fact amplitude modulated [56]. In this case, considering the modulation waveform field $B_{m}(t)$ and the carrier waveform $B_{c}(t)$ :

$$
\begin{gathered}
B_{m}(t)=M \cos \left[2 \pi f_{m} t\right] \\
B_{c}(t)=A \cos \left[2 \pi\left(2 f_{0}\right) t\right]
\end{gathered}
$$

where $M$ and $A$ are the amplitudes of the modulation (i.e., low-frequency field accounting already for the gain factor introduced by the MFCs) and carrier wave (originated by the MEMS resonance), respectively. The magnetic field reaching the sensor $\left(B_{S}(t)\right)$ results from the amplitude modulation of these signals $[66,67]$, obtained from multiplying the carrier waveform by the quantity $\left[1+B_{m}(t)\right]$ :

$$
\begin{gathered}
B_{S}(t)=\left[1+M \cos \left[2 \pi f_{m} t\right]\right] \cdot A \cos \left[2 \pi\left(2 f_{0}\right) t\right] \\
=A \cos \left[2 \pi\left(2 f_{0}\right) t\right]+\frac{1}{2} A \cdot M\left\{\cos \left[2 \pi\left(2 f_{0}-f_{m}\right) t\right]+\cos \left[2 \pi\left(2 f_{0}+f_{m}\right) t\right]\right\}
\end{gathered}
$$

Equation (9) shows that $B_{S}(t)$ (already modulated) encloses three components: the carrier waveform $B_{c}(t)$ which remains unchanged, and two sidebands at frequencies $2 f_{0} \pm f_{m}$. Therefore, it is possible to detect the low frequency magnetic field $\left(f_{m}\right)$ in the high-frequency regime $\left(2 f_{0}\right)$ by reading the output voltage of the sidebands $2 f_{0}-f_{m}$ and $2 f_{0}+f_{m}$, which can be easy understood calculating the Fourier transform of $B_{S}(t)[66,67]$ :

$$
\mathcal{F}\left(B_{s}\right)=A \delta\left(2 f_{0}\right)+\frac{1}{2} A \cdot M \delta\left(2 f_{0}-f_{m}\right)+\frac{1}{2} A \cdot M \delta\left(2 f_{0}+f_{m}\right)
$$

showing a delta-Dirac function at $2 f_{0}$ and at the two sidebands frequencies.

However, when the MEMS flaps are over the sensor element (not in the same plane) [55,58-60], one cantilever mechanical cycle corresponds to a single magnetic field cycle. Therefore, with this geometry, the modulation of a static magnetic field occurs at the same frequency of the MEMS periodic motion $\left(f_{0}\right)$. Similar calculations replacing Equation (8) by $B_{c}(t)=A \cos \left[2 \pi f_{0} t\right]$ indicate that a quasi-DC signal can be recovered from the sidebands $f_{0} \pm f_{m}$ in the modulation process.

The MEMS modulation efficiency ( $e_{\text {MEMS }}$ ) is proportional to the cantilever's mechanical displacement, increasing for larger deflection amplitudes. $e_{\text {MEMS }}$ is defined as the ratio between the amplitudes of the modulation and carrier waveforms [56], translating the carrier amplitude variation relative to its unmodulated level. A maximum efficiency $e_{\mathrm{MEMS}}=100 \%$, corresponds to a maximum amplitude change, representing a full amplitude modulation and the highest possible SNR. In practice, several factors may affect the device performance, thus reducing $e_{\text {MEMS }}$. One design/fabrication feature, which directly affects $e_{\text {MEMS }}$ is the distance between MFCs and MR sensor, which must be the smallest possible to ensure a better modulation efficiency.

Other important parameter to characterize the MEMS resonators is the quality factor (Q) [68], which quantifies how under-damped is the oscillating motion and characterizes its frequency bandwidth. A high $Q$ is associated to large amplitude stable deflections with an accurate oscillation frequency, indicating small energy dissipation. The friction induced by the physical medium where the resonator operates is the main limiting factor of $Q$. A vacuum packaging is a reliable alternative to minimize the resonator damping motion $[40,69]$.

\section{Towards picoTesla Field Detection}

Although the use of hybrid MEMS flux concentrators devices with integrated MR sensors seems to be a promising technique for the detection of ultra-low intensity magnetic fields ( $\mathrm{pT} / \mathrm{Hz}^{1 / 2}$ range), this strategy (tens of $\mathrm{kHz}$ ) has been validated only by few research groups [40,41,54-60]. The successful 
merging of MR and MEMS technology represents a great technical challenge. The combination of state-of-the-art high sensitive and low-noise MR sensors with the required steps to micro-fabricate and release the MEMS structures reveals to be a critical aspect in the development of these hybrid devices and is a limiting factor for the efficiency of this modulation technique. An overview of the results reported by five different groups is discussed in the following focusing on key points as geometries and operating conditions culminating with achieved magnetic field detection. Then on Section 3.5 the fabrication challenges for these devices are discussed.

\subsection{Approach I: MR Sensors, Coupled Capacitive MEMS with MFCs}

The MEMS flux concentrator developed by Edelstein et al. [40,41] consisted of a SV sensing element (active area: $3 \times 88 \mu^{2}$ ) placed between two Si cantilever resonators. These were fabricated on silicon-on-insulator (SOI) wafers and incorporating trapezoidal bilayers of $\mathrm{Cr} / \mathrm{Ni}_{80} \mathrm{Fe}_{20}$ [70] acting as MFCs on top. The height of the trapezoid was set in $104 \mu \mathrm{m}$, while the pole and yoke had a width of 80 and $150 \mu \mathrm{m}$, respectively, corresponding to a field amplification of 2 (MFCs gap $=52 \mu \mathrm{m}$ ). The reduced MFCs size is required to ensure that the MEMS resonates in the desired high frequency band. Due to non-uniformity in the MEMS fabrication process, a dispersion in the resonance frequency of the two cantilevers occurs. Then, besides displaying oscillations not entirely in phase, the relative phase also tends to drift. To overcome this problem, polysilicon springs ( $4 \mu \mathrm{m}$ wide) connecting the two MEMS flaps were incorporated in the design, inducing a mechanical coupling which forces a common normal-mode resonance frequency. Figure 6 shows a schematic view of the developed MEMS flux concentrator design and a SEM top view of the fabricated device. With this design a $180^{\circ}$ out of phase motion of the MEMS flaps corresponds to a higher resonance frequency (second normal resonance mode) when compared with the in-phase oscillating motion (first normal resonance mode). The resonant vibration mode is induced by electrostatic comb drives introduced in the design.
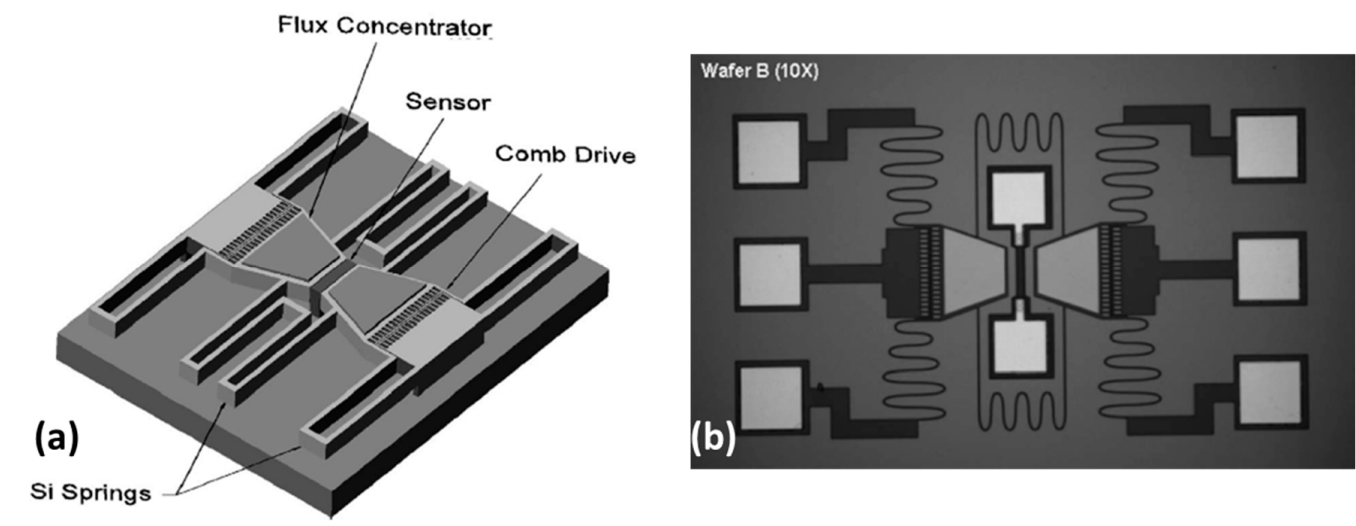

Figure 6. (a) Schematic view and (b) SEM image of the proposed MEMS flux concentrator device. Reprinted with permission from Edelstein A.S. et al., J. Appl. Phys. 2006, 99, 08B317. Copyright 2006 AIP Publishing LLC.

This structure is characterized by a resonance frequency $f_{0} \approx 24 \mathrm{kHz}$ and a corresponding quality factor $Q \approx 30$ [54]. Validation was achieved by shifting magnetic signals with a frequency of $25 \mathrm{~Hz}$ and intensities of $22 \mu \mathrm{T}$ and $1.3 \mu \mathrm{T}$ to the high frequency range (around $48 \mathrm{kHz}$ ) and recording the respective sidebands. The resonator flaps were driven into an in-phase oscillating motion, exhibiting a maximum vertical deflection of $5 \mu \mathrm{m}$.

\subsection{Approach II: MR Sensors, Capacitive MEMS (Single Cantilever and Torsional Paddle) with MFCs}

To modulate a low-intensity and low-frequency magnetic field into the high frequency range, INESC-MN explored two different hybrid MEMS flux concentrator devices: (i) SV sensor with static 
MFCs and a single MFC-MEMS cantilever [55]; and (ii) a MTJ sensor placed under a MEMS torsional structure with both static and oscillating MFCs [57].

In the first approach, a single SV sensor (active area: $10 \times 2 \mu \mathrm{m}^{2}$ ) is patterned together with two static trapezoidal MFCs (350 nm thick amorphous CoZrNb alloy), presenting a gap of $3.5 \mu \mathrm{m}$ [55]. A micro-machined MEMS cantilever resonator of hydrogenated amorphous silicon ( $400 \mathrm{~nm}$ thick, $30 \times 16 \mu \mathrm{m}^{2}$ area) with an air gap of $1.5 \mu \mathrm{m}$ between its bottom surface and the gate electrode is fabricated as close as possible to one of static MFCs (separation of $1 \mu \mathrm{m}$ ). The gate electrode consists of a $200 \mathrm{~nm}$ thick Al lead placed under the cantilever structure, while a thinner Al layer (100 $\mathrm{nm}$ thick) is deposited on the cantilever surface to act as counter-electrode for electrostatic actuation. An additional MFC (CoZrNb $200 \mathrm{~nm}$ ) is deposited and patterned on top of the cantilever. The characterization of this combined MFCs structures results in a field gain of 5 times. A SEM image of the fabricated device and a cross section schematic view are presented in Figure 7.
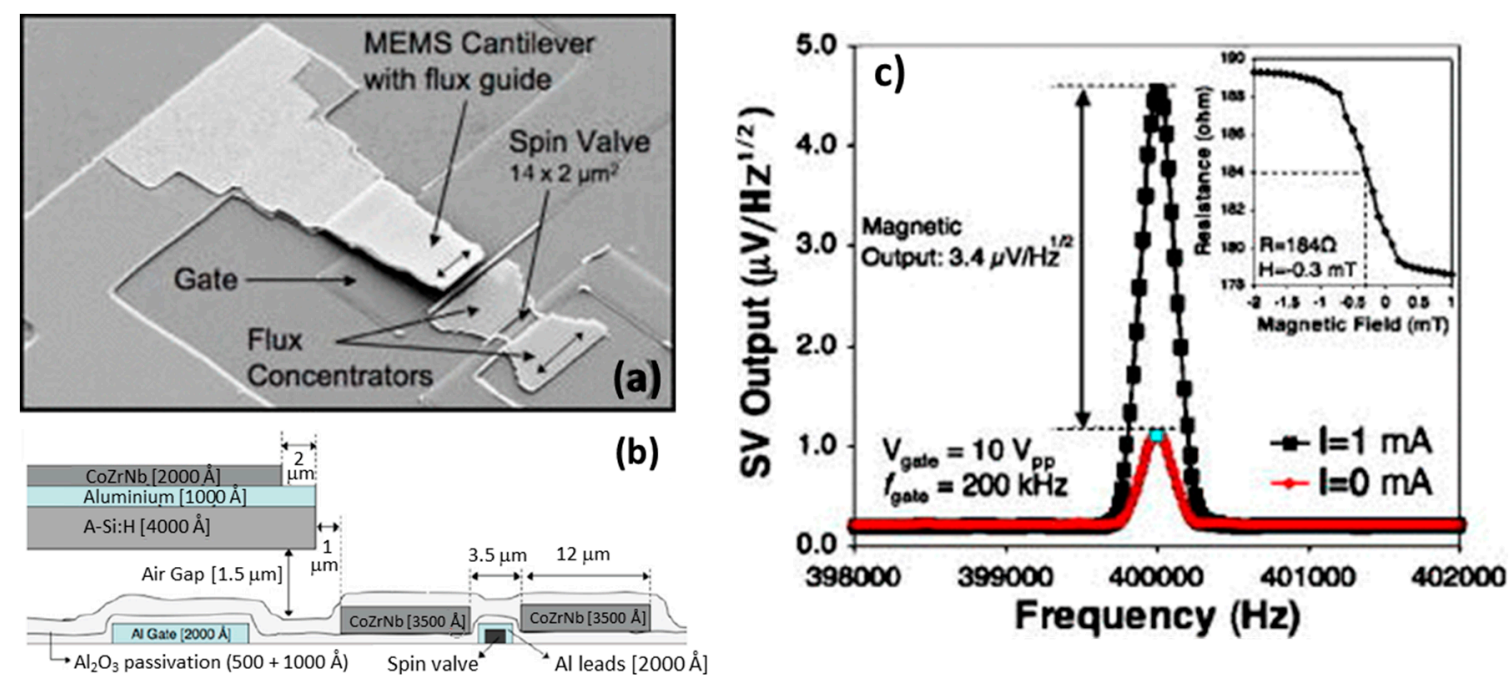

Figure 7. (a) SEM image of the hybrid device showing the integration of a SV sensor with static MFCs and a single MEMS cantilever resonator (with an additional MFC on top); (b) Schematic view of the device cross section; (c) SV voltage output exhibiting both electric (capacitive coupling) and magnetic components when the cantilever resonates at a frequency of $200 \mathrm{kHz}$. Reprinted with permission from Guedes A. et al., J. Appl. Phys. 2008, 103, 07B924. Copyright 2008 AIP Publishing LLC.

Applying an AC signal with an amplitude of $10 \mathrm{~V}_{\mathrm{pp}}$ and a frequency of $200 \mathrm{kHz}$ to the device gate electrode, makes the cantilever oscillate at $400 \mathrm{kHz}$, being able to modulate a DC external magnetic field of $0.3 \mathrm{mT}$. The SV sensor output (bias current: $1 \mathrm{~mA}$ ) has an electric component coming from the direct AC coupling with the gate electrode $\left(1.2 \mu \mathrm{V} / \mathrm{Hz}^{1 / 2}\right)$ and a pure magnetic contribution $\left(3.4 \mu \mathrm{V} / \mathrm{Hz}^{1 / 2}\right)$, visible in Figure 7c. However, this particular device shows a low modulation efficiency from DC to AC $\left(e_{\text {MEMS }}=0.11 \%\right)$, so the improvement by operating the sensor at its thermal background is mitigated by signal losses due to low $e_{\text {MEMS }}$. A minimum detectable static field of $540 \mathrm{nT} / \mathrm{Hz}^{1 / 2}$ is reported.

A different approach consists that uses a MEMS torsional structure (width $20 \mu \mathrm{m}$ ) fabricated by surface micromachining with hydrogenated amorphous silicon ( $300 \mathrm{~nm}$ thick). The sensing element is now a MgO MTJ sensor (pillar area: $15 \times 15 \mu^{2}$ ) placed under the torsionator [57]. A single 400 $\mathrm{nm}$ thick CoZrNb static MFC was patterned close to the MTJ, while an additional $200 \mathrm{~nm}$ thick layer of this soft material was deposited and patterned on top of the MEMS torsional structure. Both gate electrode and counter electrode were defined as in the first approach. Figure 8 shows a SEM image of the fabricated hybrid device and a schematic view of its cross section. This device was used for DC magnetic field modulation and detection at high frequencies, in an attempt to improve the MEMS modulation efficiency and the minimum detectable field. Figure $8 \mathrm{c}$ shows the sensor voltage output 
when an AC signal of $20 \mathrm{~V}_{\mathrm{pp}}$ and a frequency of $230 \mathrm{kHz}$ is applied to the device gate electrode. The field detected by the MTJ sensor is modulated at $460 \mathrm{kHz}$ as a result of the torsional bridge resonating motion. The difference between the results obtained for the sensor operating in the linear range or at saturation corresponds to the magnetic component $\left(\sim 240 \mu \mathrm{V} / \mathrm{Hz}^{1 / 2}\right)$. The device's $e_{\text {MEMS }}$ (from DC to AC) is about $11 \%$. This $e_{\text {MEMS }}$ enhancement reflects the higher deflection amplitude achieved by this type of structures when compared to resonator cantilevers. For this operating condition, the DC field detection limit is $40 \mathrm{nT} / \mathrm{Hz}^{1 / 2}$, improving by about one order of magnitude compared to the initial situation characterized by a low $e_{\text {MEMS }}$ (detection level of $540 \mathrm{nT} / \mathrm{Hz}^{1 / 2}$ ).
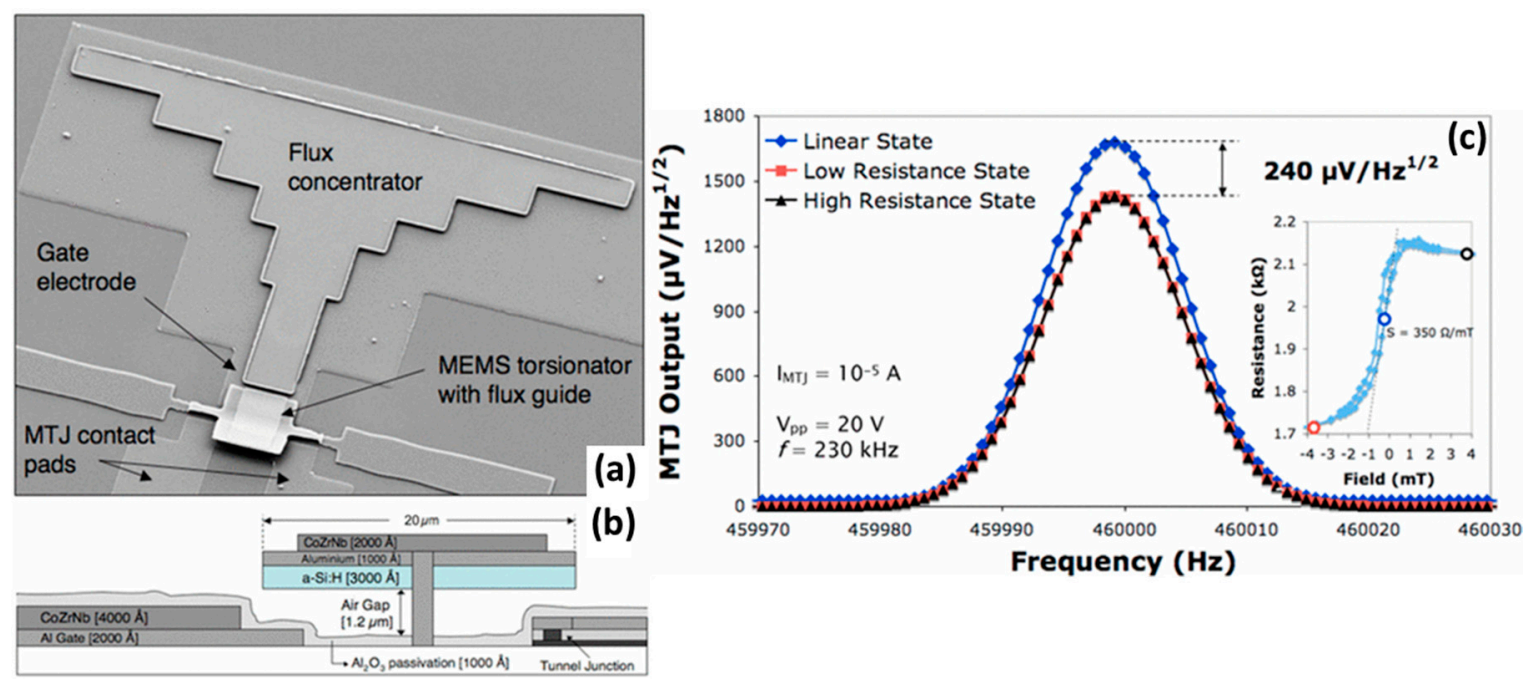

Figure 8. (a) SEM image of the device exhibiting the MEMS torsional structure integrated with a MTJ sensor and both static/dynamic MFCs; (b) Schematic view of the device cross section; (c) MTJ voltage output corresponding to the detection of a DC magnetic field of $0.36 \mathrm{mT}$ when the torsional structure is actuated at a frequency of $230 \mathrm{kHz}$. Both magnetic and electric (capacitive coupling) components are presented. Reproduced with permission from Guedes A. et al., IEEE Trans. Magn. 2008, 44, 2554; published by IEEE, 2008.

\subsection{Approach III: MR Sensors, Piezoelectric MEMS with MFCs}

Another successful example of such a hybrid device integrates SVs and piezoelectric MEMS cantilevers. Piezoelectric MEMS devices have a number of advantages over electrostatic MEMS presented above. They require lower driving voltage ( one order of magnitude for PZT based cantilevers [71]), are more area efficient and do not require air-gap capacitors with complex geometries and exposed conductors. The principle of operation of piezoelectric cantilevers is based on the property of the active material employed in its manufacturing-piezoelectricity. Piezoelectric materials generate an electric voltage when subjected to a mechanical deformation. One of the unique characteristics of this phenomenon is its reversibility; materials exhibiting the direct effect of piezoelectricity will also experience the inverse effect, a mechanical deformation when an electric field is applied. One drawback of piezoelectric MEMS is the more complex fabrication process when compared with capacitive MEMS. However, from a performance point of view, piezoelectric MEMS are more attractive [71].

Although employing a different driving mechanism for the MEMS cantilevers, this approach has a design similar to the one proposed by Edelstein et al [40,41]. Single SV element (active area: $\left.40 \times 1.5 \mathrm{~mm}^{2}\right)$ is placed in the gap of two SOI/Mo $(100 \mathrm{~nm}) / \mathrm{AlN}(700 \mathrm{~nm})$ piezoelectric MEMS cantilevers with patterned MFCs on top [56]. Figure 9a displays a SEM picture of the fabricated hybrid device, depicting the SV sensor suspended on a bridge centered in the gap between the two AlN-based piezoelectric cantilevers. In this case, multilayers of antiferromagnetic coupled $\mathrm{CoFeB}$ layers were used as MFCs ([CoFeB $38 \AA / \mathrm{Ru} 18 \AA]$ × 32/CoFeB $38 \AA$ ). The cantilever resonator structures were 
patterned with a fixed width of $40 \mu \mathrm{m}$, and different lengths ranging from 200 to $400 \mu \mathrm{m}$. The larger cantilever structure has a resonance frequency $f_{0}=17.7 \mathrm{kHz}$, a quality factor $Q=181$, and $e_{\mathrm{MEMS}}=1.6 \%$. Figure $9 \mathrm{~b}$ shows the sidebands in the high frequency range obtained from the modulation of an $0.3 \mathrm{mT}$ external magnetic field with a frequency $f_{m}=30 \mathrm{~Hz}$, upon a cantilever actuation with $1.4 \mathrm{~V}_{\mathrm{rms}}$ at $f_{0}$. The minimum detectable field of the device is $301 \mathrm{nT} / \mathrm{Hz}^{1 / 2}$ for the detection of DC magnetic fields, and $602 \mathrm{nT} / \mathrm{Hz}^{1 / 2}$ for low frequency fields (taking into account the $1 / 2$ loss from the modulated high-frequency sidebands).
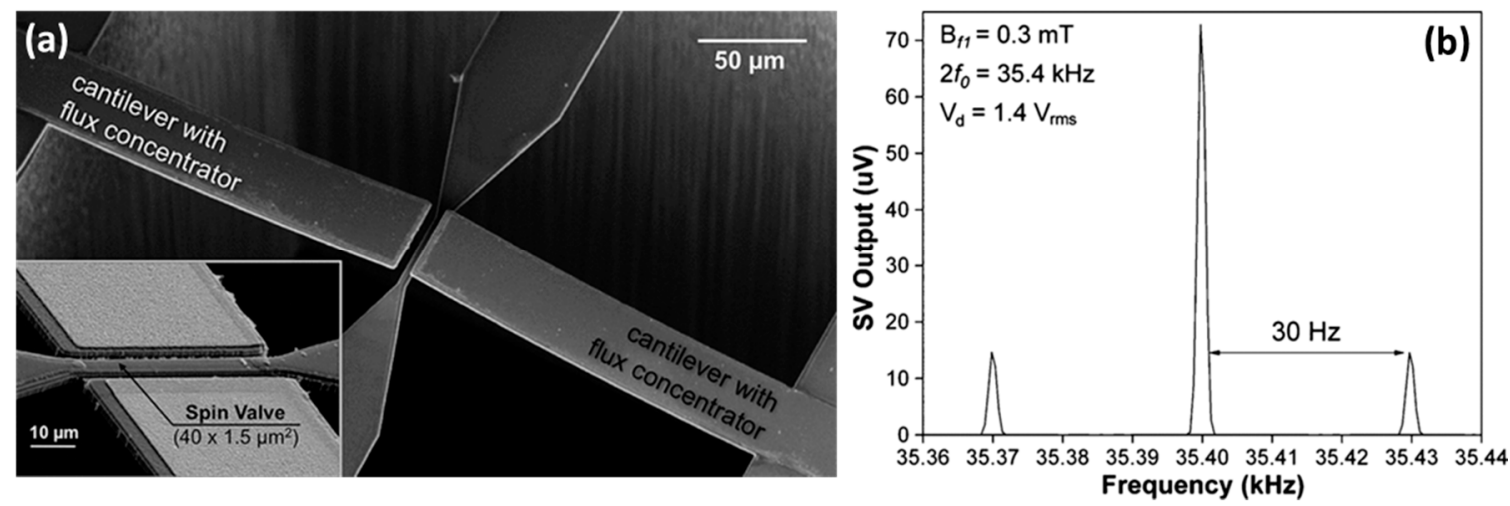

Figure 9. (a) SEM image of the developed device, showing the SV sensing element between two resonator cantilevers with integrated MFCs; (b) Acquired data resulting from the modulation of a low-frequency AC field $(0.3 \mathrm{mT}$ with a frequency of $30 \mathrm{~Hz})$, exhibiting the respective sidebands when the cantilevers are actuated at the resonance frequency $\left(f_{0}=17.7 \mathrm{kHz}\right)$. Reproduced with permission from Guedes A. et al., IEEE Trans. Magn. 2012, 48, 4115; published by IEEE, 2012.

A parallel approach based on vertical motion flux modulation (VMFM) was also targeting $e_{\text {MEMS }}$ enhancement [58-60]. In this case, a micron size piezoelectric Si cantilever is suspended above the gap between a pair of MFCs. Commercial giant-magnetoresistive (GMR) sensors in a bridge configuration are used and placed between the MFCs (gap $=60 \mu \mathrm{m})$. The MEMS structure is composed of a Si cantilever with a piezoelectric ceramic (lead zirconium titanate-PZT, top surface) and a soft magnetic film (bottom surface). The latter is an electroplated $\mathrm{Ni}_{79} \mathrm{Fe}_{21}$ layer with $8 \mu \mathrm{m}$ thick and dimensions of $600 \mu \mathrm{m} \times 120 \mu \mathrm{m}$, being suspended at a static height of about $10 \mu \mathrm{m}$. These dimensions ensure that it covers the MFCs gap and thus acts as a flux modulation film as it is driven vertically by the MEMS actuator. When the cantilever approaches the MFCs gap, the magnetic flux goes preferentially through the NiFe film, decreasing the magnetic field that reaches the GMR elements. On the other hand, when the cantilever moves upwards the magnetic field in the gap region is restored. Figure 10a,b show a schematic operating view of the device. Its validation was achieved upon the modulation of a $1.2 \mu \mathrm{T}$ AC magnetic field with a frequency of $1 \mathrm{~Hz}$. The obtained output power spectrum is shown in Figure 10c, where the two high frequency sidebands are observed. The cantilever structure oscillates under a resonance frequency $f_{0}=3.57 \mathrm{kHz}$, reaching a displacement amplitude of $8 \mu \mathrm{m}$ (in the air) under an AC driving voltage $(6 \mathrm{~V})$ which corresponds to a $Q \approx 14$. The $e_{\text {MEMS }}$ of this device is about $19 \%$. The shift in the sensor operating point to the high frequency range causes a noise level reduction around $18 \times$, corresponding to a DC minimum detectable filed of $530 \mathrm{pT} / \mathrm{Hz}^{1 / 2}$. 


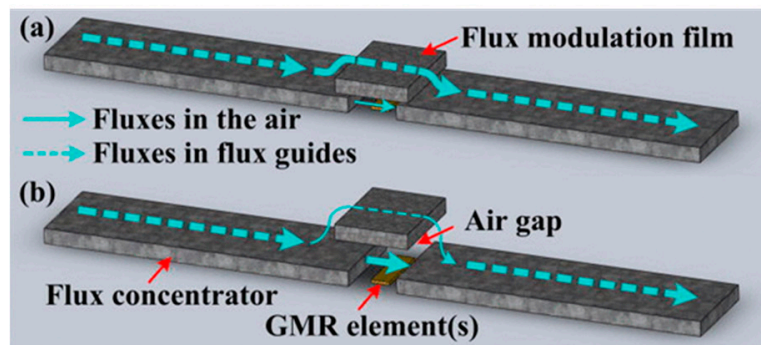

GMR element(s)

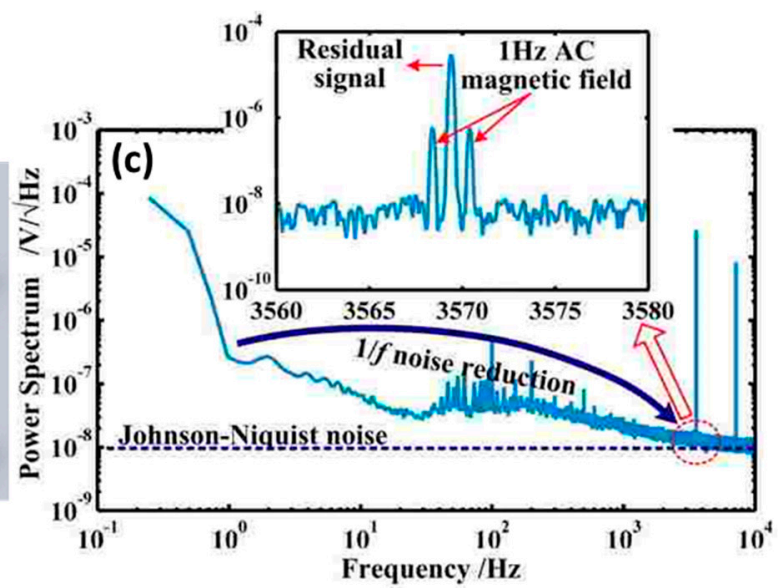

Figure 10. Schematic operating view of the VMFM based device: (a) cantilever gets close the sensing elements, reducing the magnetic flux through them; (b) cantilever moves upwards, restoring the magnetic flux in the sensing elements; (c) Acquired device output resulting from the modulation of a low-frequency AC field $(1.2 \mu \mathrm{T}$ with a frequency of $1 \mathrm{~Hz})$, exhibiting the respective sidebands when the cantilevers are actuated at the resonance frequency $\left(f_{0}=3.57 \mathrm{kHz}\right)$. The residual signal at $f_{0}$ arises from the remanence of the $\mathrm{Ni}_{79} \mathrm{Fe}_{21}$ flux modulation field and/or from an electric coupling between then sensing elements and the MEMS piezoelectric structure. Reprinted with permission from Hu J. et al., Appl. Phys. Lett. 2012, 100, 244102. Copyright 2012 AIP Publishing LLC.

\subsection{Approach IV: MR Sensors, Bulk-Micromachined MEMS with MFCs}

A final example resorts to in-plane, bulk-micromachined electrostatic actuators combined with SV sensors and MFCs. The modulation of the magnetic field sensed by the SVs is achieved by having part of the MFC patterned atop a large stroke micro actuator. Such actuators are obtained by standard micromachining, deep reactive ion etching (DRIE) of silicon-on-insulator (SOI) substrates [72]. When compared to other types of MEMS devices (e.g., surface micromachined structures [53,73]), larger displacements can be achieved by a simple design of the movable and anchor parts, dimensioning of the inertial mass, parallel flexures and interdigitated comb drives [74]. Larger modulation effects can therefore be expected, at the expense of lower resonance frequencies [74,75].

Figure 11a,b shows images of a fabricated device with SV sensors (active area: $2 \times 20 \mu \mathrm{m}^{2}$ ) and MFCs of $400 \mathrm{~nm}$ thick CoZrNb [57]. The MEMS are actuated applying an AC voltage with DC component to the comb drive (electrostatic driving), responsible for a motion at $f_{0}$ and $2 f_{0}$. The actuator has $f_{0}=7.5 \mathrm{kHz}$ and vibration amplitude of $17.5 \mu \mathrm{m}$. Despite such large displacement, it only reaches an efficiency $e_{\text {MEMS }}=1.03 \%$, comparable to that of the modulator described in [56]. This low $e_{\text {MEMS }}$ value is attributed to the larger gaps used in the bulk micromachined device. The detectivity is around $80 \mathrm{nT} / \mathrm{Hz}^{1 / 2}$ for quasi-DC magnetic fields $(7 \mathrm{~Hz}$ ), as can be extracted from Figure 11c. A simulation of the device illustrating the magnetic flux concentration in the sensing elements is presented in Figure 11d, highlighting the minimum (off) and maximum (on) concentration positions. Current work to increase the efficiency of these devices focus on further increasing the actuators displacements above $100 \mu \mathrm{m}$, reducing the separation between MFCs and SVs, optimizing the concentration on/off (i.e., design layout and improved materials) and increasing their resonance frequency targeting a reduction of the inertial mass alongside an increase of the spring constants but without compromising displacement. 

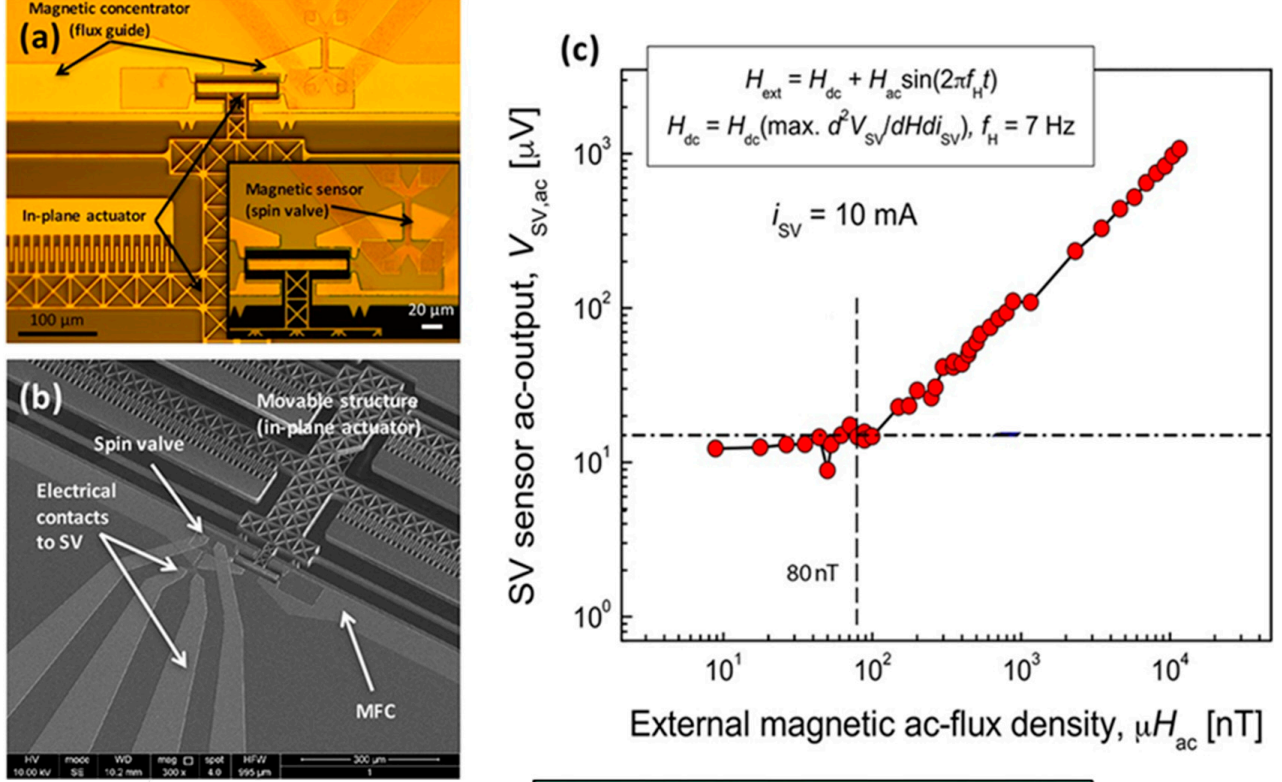

(d)

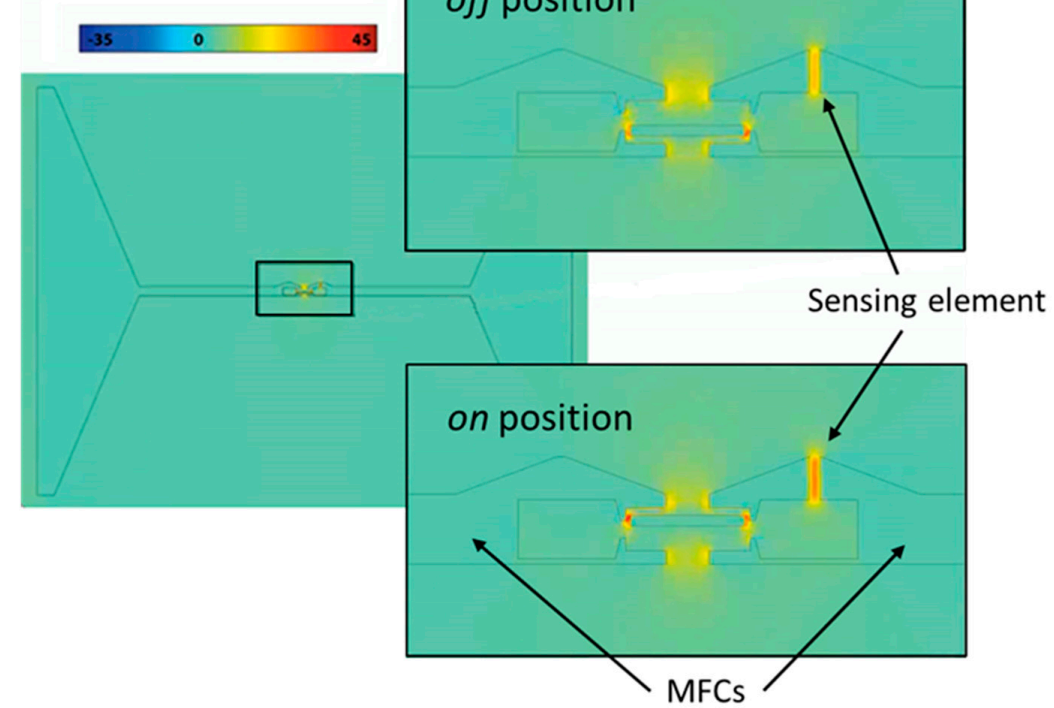

Figure 11. (a) Optical and (b) SEM image of modulator combining in-plane electrostatic microactuators with interdigitated fingers comb drive, SV sensors and MFCs; (c) Modulated sensor output as a function of magnetic field amplitude at low frequency $(7 \mathrm{~Hz})$; (d) Finite element simulation which illustrates the concentrated magnetic flux in the sensing element for the two positions: off-minimum concentration; on-maximum concentration.

\subsection{Fabrication Details on MEMS Integration with MR Sensors}

The exchange bias in the AFM/FM interface of MR sensors is set by magnetic annealing. Consequently, the thermal stability of the sensing elements must be taken into account during its fabrication process (e.g., ion milling etch, metallization, passivation), ensuring a working temperature $<150^{\circ}$. The standard fabrication process of the described hybrid technologies starts with the microfabrication of MR sensors, being followed by the definition of the MEMS structures. Therefore, after being patterned the sensing elements are subjected to conditions, which can affect both their magnetic and physical properties. However, these conditions (e.g., high temperature, presence of high reactive chemical etchants) are required for the MEMS fabrication, which comprises its geometry definition, electrodes deposition and structure release. 
A common technique to remove the sacrificial layer (typically $\mathrm{SiO}_{2}$ ) holding the resonator consists in the use of vapor HF [76]. The major drawback of this technique is the consequent physical damage/corrosion of the MR structure. To avoid the presence of such reactive solution, oxygen plasma can be used as an alternative removal method. The latter does not affect the physical integrity of the sensing element, however the required high temperatures are an issue not only for the sensor magnetic response but also for the magnetic behavior of the MFCs materials. A final annealing step to recover the sensor magnetic response is not a viable solution since the magnetic properties of the MFCs would not be recovered, compromising the obtained flux gain.

In the approaches using SOI wafers, a backside micromachining of the wafer is required to remove the bottom Si (thickness of few $\mu \mathrm{m}$ ) until the sacrificial layer. However, due to the large aspect ratio of the intended cavities, the DRIE on SOI wafers leads to silicon grass formation [77] and consequently changes the MEMS physical properties (e.g., heavier resonator with different $f_{0}$ ). To overcome this problem, a wafer backside grinding can be performed reducing the cavities aspect ratio.

\section{Final Remarks}

This paper reviews the performance of hybrid devices resulting from the integration of MR sensors with MEMS resonators, in an attempt to detect DC or low-frequency external magnetic fields through their mechanical modulation. The latter consequently shifts the signal to the high frequency range due to the incorporation of MFCs on MEMS. This merging technology allows the MR sensor to operate at high frequencies, overcoming the higher noise spectrum density present at low frequencies as a result of the $1 / f$ component.

Table 2 summarizes the results obtained for the performance of the different MEMS flux concentrator strategies presented in this review, highlighting the minimum detectable field, operation frequency and modulation efficiency. The integration of a MTJ sensor with the MEMS torsional structure [57] proved to be a reliable alternative, allowing an enhancement of static DC field detection limit from hundreds to tens of $\mathrm{nT} / \mathrm{Hz}^{1 / 2}$, mainly due to the increase in the modulation efficiency to $11 \%$. The larger mechanical deflections of MEMS torsional structures and the use of a sensing element with higher signal can be on the base of the observed improvement. Furthermore, the suspension of a piezoelectric MEMS above GMR sensors placed in the gap of two static MFCs [58-60] provided an enhancement of the minimum detectable field to hundreds of $\mathrm{pT} / \mathrm{Hz}^{1 / 2}$, alongside with a modulation efficiency of 19\%. However, single MTJ sensors with incorporated static MFCs (no MEMS) demonstrated a better performance at low-frequencies $(\sim 100 \mathrm{~Hz})$, where minimum detectable fields around tens of $\mathrm{pT} / \mathrm{Hz}^{1 / 2}$ were reported $[10,12]$. The lower detection levels obtained for non-integrated MR sensors combined with its simpler fabrication process make them a more reliable alternative than hybrid MR-MEMS in terms of performance and fabrication.

The presented results underline the great potential of MR-MEMS hybrid devices in the detection of low-intensity and low-frequency external magnetic fields with a wide room for improvement. However, the application of the described devices for this purpose is limited by two main factors: (i) the MR sensor's sensitivity and (ii) the effective $1 / f$ noise suppression. The latter is achieved by the field modulation employed by the MEMS structures and quantified by the modulation efficiency factor $\left(e_{\mathrm{MEMS}}\right)$. An insightful understanding of the aspects limiting the overall performance of the aforementioned hybrid devices is crucial in the development of an optimized strategy that would pave the way towards the detection of picoTesla. In terms of sensitivity, a relevant improvement is obtained with the inclusion of MFCs as shown in Section 2.3, where major optimizations on this front have been done with proven results. In addition, although MR sensors based on MTJs have a higher noise level compared with SV elements, their higher SNR leads to an enhanced detection limit, justifying the choice of MTJs when targeting picoTesla detection despite the extra complexity of the fabrication process. Regarding the drive mechanisms of MEMS structures, the approaches based on capacitive MEMS [55,57] reported the disruption of some of the MR sensors caused by the gate electrode high voltage, leading to low yield of final devices. In this scope, piezoelectric MEMS 
cantilevers appear as a strong alternative. As $e_{\text {MEMS }}$ is directly related with the amount of external field that MEMS flux concentrators are capable of capture and thus modulate, a key aspect to improve it consists in the minimization of the distance between MEMS and sensing element. In this context, the geometry based on a pair of cantilevers [56] reveals as a promising approach, since the magnetic field is captured from both sides of the MR sensor. Since the sensor output varies accordingly with the MEMS oscillating motion, the mechanical displacement of the MEMS structure is also intrinsically

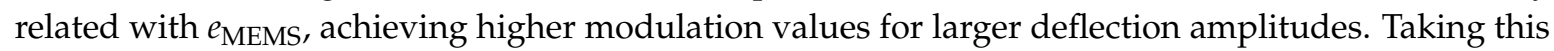
into account, the geometry and dimensions of the MEMS structures must be carefully chosen aiming a larger deflection capability. Long and narrow cantilevers can achieve large displacements at the expense of an increased device footprint. Envisaging applications requiring a small device footprint, the use of materials with different mechanical properties must be address to achieve larger deflections with small dimension resonators.

The optimization of $e_{\text {MEMS }}$ to values $~ 50 \%$ by using MEMS structures characterized by larger deflection amplitudes together with a MFCs gain improvement arising from a tapered profile $(\sim 100 \times)$ may allow the fabricated hybrid devices to reach detection levels in the $\mathrm{pT} / \mathrm{Hz}^{1 / 2}$ range, placing these devices at the forefront of low-intensity and low-frequency magnetic fields detection.

Table 2. Comparison of the different proposed hybrid devices integrating MR sensors with MEMS flux concentrators regarding the modulation efficiency and minimum detectable field.

\begin{tabular}{|c|c|c|c|c|}
\hline Used Strategy & MR Sensor & $\begin{array}{c}\text { Modulation } \\
\text { Frequency (kHz) }\end{array}$ & $\begin{array}{l}\text { Modulation } \\
\text { Efficiency (\%) }\end{array}$ & $\begin{array}{c}\text { Minimum Detectable } \\
\text { Field }\left(\mathrm{nT} / \mathrm{Hz}^{1 / 2}\right)\end{array}$ \\
\hline $\begin{array}{l}\text { INESC-MN \#1: capacitive } \\
\text { single cantilever [55] }\end{array}$ & SV & 400 & 0.11 & 540 (DC) \\
\hline $\begin{array}{l}\text { Picosense/INESC-MN: piezoelectric } \\
\text { paired cantilevers [56] }\end{array}$ & SV & 35.4 & 1.6 & $\begin{array}{l}301(\mathrm{DC}) \\
602(\mathrm{AC})\end{array}$ \\
\hline $\begin{array}{l}\text { INL/INESC-MN: bulk micromachined } \\
\text { electrostatic actuator }\end{array}$ & SV & $\begin{array}{l}7.5 \\
15\end{array}$ & 1.03 & $80(\mathrm{AC})$ \\
\hline
\end{tabular}

Acknowledgments: This work was supported by FCT-project EXCL/CTM-NAN/0441/2012. João Valadeiro would like to thank Diana C. Leitao for her comments on the manuscript and acknowledges FCT for scholarship grant PD/BD/113956/2015. INESC-MN acknowledges funding from Pest-OE/CTM/LA0024/2011.

Author Contributions: Paulo P. Freitas, Andre Guedes and João Gaspar conceived and designed the experiments. Andre Guedes and João Gaspar microfabricated the MEMS and João Valadeiro, Rita Macedo and Susana Cardoso microfabricated the magnetic devices. All authors participated in the characterization of the devices and data analysis. João Valadeiro wrote the paper with contributions from all authors.

Conflicts of Interest: The authors declare no conflict of interest.

\section{References}

1. Lenz, J.; Edelstein, A. Magnetic Sensors and Their Applications. IEEE Sens. J. 2006, 6, 631-649. [CrossRef]

2. Jogschies, L.; Klaas, D.; Kruppe, R.; Rittinger, J.; Taptimthong, P.; Wienecke, A.; Rissing, L.; Wurz, M. Recent Developments of Magnetoresistive Sensors for Industrial Applications. Sensors 2015, 15, 28665-28689. [CrossRef] [PubMed]

3. He, D.F.; Tachiki, M.; Itozaki, H. Highly sensitive anisotropic magnetoresistance magnetometer for eddy-current nondestructive evaluation. Rev. Sci. Instrum. 2009, 80, 036102. [CrossRef] [PubMed]

4. Freitas, P.P.; Cardoso, F.A.; Martins, V.C.; Martins, S.; Loureiro, J.; Amaral, J.; Chaves, R.C.; Cardoso, S.; Germano, J.; Piedade, M.S.; et al. Spintronic platforms for biomedical applications. Lab Chip 2012, 12, 546-557. [CrossRef] [PubMed]

5. Pannetier-Lecoeur, M.; Fermon, C.; Le Goff, G.; Simola, J.; Kerr, E. Femtotesla magnetic field measurement with magnetoresistive sensors. Science 2004, 304, 1648-1650. [CrossRef] [PubMed] 
6. Smith, N.; Jeffers, F.; Freeman, J. A High-Sensitivity Magnetoresistive Magnetometer. J. Appl. Phys. 1991, 69, 5082-5084. [CrossRef]

7. Damsgaard, C.D.; Freitas, S.C.; Freitas, P.P.; Hansen, F.M. Exchange-biased planar Hall effect sensor optimized for biosensor applications. J. Appl. Phys. 2008, 103, 07A302. [CrossRef]

8. Tsymbal, E.; Pettifor, D.G. Perspectives of giant magnetoresistance. Solid State Phys. 2001, 56, $113-237$.

9. Heim, D.E.; Fontana, R.E.; Tsang, C.; Speriosu, V.S.; Gurney, B.A.; Williams, M.L. Design and operation of spin valve sensors. IEEE Trans. Magn. 1994, 30, 316-321. [CrossRef]

10. Chaves, R.; Freitas, P.P.; Ocker, B.; Maass, W. Low frequency picoTesla field detection using hybrid MgO based tunnel sensors. Appl. Phys. Lett. 2007, 91, 102504. [CrossRef]

11. Paz, E.; Serrano-Guisan, S.; Ferreira, R.; Freitas, P.P. Room temperature direct detection of low frequency magnetic fields in the $100 \mathrm{pT} / \mathrm{Hz}^{0.5}$ range using large arrays of magnetic tunnel junctions. J. Appl. Phys. 2014, 115, 17E501. [CrossRef]

12. Cardoso, S.; Leitao, D.C.; Gameiro, L.; Cardoso, F.; Ferreira, R.; Paz, E.; Freitas, P.P. Magnetic tunnel junction sensors with pTesla sensitivity. Microsyst. Technol. 2014, 20, 793-802. [CrossRef]

13. Dieny, B. Giant magnetoresistance in spin-valve multilayers. J. Magn. Magn. Mater. 1994, 136, 335-359. [CrossRef]

14. Cardoso, S.; Gehanno, V.; Ferreira, R.; Freitas, P.P. Ion Beam Deposition and oxidation of Spin Dependent Tunnel Junctions. IEEE Trans. Magn. 1999, 35, 2952-2954. [CrossRef]

15. Yuasa, S.; Djayaprawira, D.D. Giant tunnel magnetoresistance in magnetic tunnel junctions with a crystalline $\mathrm{MgO}(001)$ barrier. J. Phys. D Appl. Phys. 2007, 40, R337. [CrossRef]

16. Freitas, P.P.; Ferreira, R.; Cardoso, S.; Cardoso, F. Magnetoresistive sensors. J. Phys. Condens. Matter 2007, 19, 165221. [CrossRef]

17. Silva, A.V.; Leitao, D.C.; Valadeiro, J.; Amaral, J.; Freitas, P.P.; Cardoso, S. Linearization strategies for high sensitivity magnetoresistive sensors. Eur. Phys. J. Appl. Phys. 2015, 72, 10601. [CrossRef]

18. Ferreira, R.; Paz, E.; Freitas, P.P.; Wang, J.; Xue, S. Large area and low aspect ratio linear magnetic tunnel junctions with a soft-pinned sensing layer. IEEE Trans. Magn. 2012, 48, 3719-3722. [CrossRef]

19. Freitas, P.P.; Cardoso, S.; Ferreira, R.; Martins, V.R.; Guedes, A.; Cardoso, F.; Loureiro, J.; Macedo, R.; Chaves, R.; Amaral, J.P. Optimization and integration of magnetoresistive sensors. J. SPIN 2011, 1, 71. [CrossRef]

20. Lei, Z.Q.; Li, G.J.; Egelhoff, W.F., Jr.; Lai, P.T.; Pong, P.W. Review of Noise Sources in Magnetic Tunnel Junction Sensors. IEEE Trans. Magn. 2011, 47, 602-612. [CrossRef]

21. Ozbay, A.; Gokce, A.; Flanagan, T.; Stearrett, R.A.; Nowak, E.R.; Nordman, C. Low frequency magnetoresistive noise in spin-valve structures. Appl. Phys. Lett. 2009, 94, 202506. [CrossRef]

22. Jiang, L.; Nowak, E.R.; Scott, P.E.; Johnson, J.; Slaughter, J.M.; Sun, J.J.; Dave, R.W. Low-frequency magnetic and resistance noise in magnetic tunnel junctions. Phys. Rev. B 2004, 69, 054407. [CrossRef]

23. Johnson, J.B. Thermal agitation of electricity in conductors. Phys. Rev. Lett. 1928, 32, 97. [CrossRef]

24. George, P.K.; Wu, Y.; White, R.M.; Murdock, E.; Tondra, M. Shot noise in low resistance magnetic tunnel junctions. Appl. Phys. Lett. 2002, 80, 682-684. [CrossRef]

25. Zhu, J.G. Thermal magnetic noise and spectra in spinvalve heads. J. Appl. Phys. 2002, 91, 7273-7275. [CrossRef]

26. Egelhoff, W.F., Jr.; Pong, P.W.T.; Unguris, J.; McMichael, R.D.; Nowak, E.R.; Edelstein, A.S.; Burnette, J.E.; Fisher, G.A. Critical challenges for picoTesla magnetic-tunnel-junction sensors. Sens. Actuators A 2009, 155, 217-225. [CrossRef]

27. Kogan, S. Electronic Noise and Fluctuations in Solids; Cambridge University Press: Cambridge, UK, 2008.

28. Han, G.C.; Zong, B.Y.; Luo, P.; Wang, C.C. Magnetic field dependence of low frequency noise in tunnel magnetoresistance heads. J. Appl. Phys. 2010, 107. [CrossRef]

29. Ren, C.; Liu, X.; Schrang, B.D.; Xiao, G. Low-frequency magnetic noise in magnetic tunnel junctions. Phys. Rev. B 2004, 69. [CrossRef]

30. Hooge, F.N.; Hoppenbrouwers, A. $1 / f$ noise in continuous gold films. Physica 1969, 45, 386-392. [CrossRef]

31. Chaves, R.C.; Cardoso, S.; Ferreira, R.; Freitas, P.P. Low aspect ratio micron size tunnel magnetoresistance sensors with permanent magnet biasing integrated in the top lead. J. Appl. Phys. 2011, 109, 07E506. [CrossRef]

32. Valadeiro, J.; Amaral, J.; Leitao, D.C.; Ferreira, R.; Cardoso, S.F.; Freitas, P.P. Strategies for pTesla field detection using magnetoresistive sensors with a soft pinned sensing layer. IEEE Trans. Magn. 2015, 51. [CrossRef]

33. Guo, D.W.; Cardoso, F.A.; Ferreira, R.; Paz, E.; Cardoso, S.; Freitas, P.P. MgO-based magnetic tunnel junction sensors array for non-destructive testing applications. J. Appl. Phys. 2014, 115, 17E513. [CrossRef] 
34. Leitao, D.C.; Paz, E.; Silva, A.V.; Moskaltsova, A.; Knudde, S.; Deepak, F.; Ferreira, R.; Cardoso, S.; Freitas, P.P. Nanoscale Magnetic Tunnel Junction sensing devices with soft pinned sensing layer and low aspect ratio. IEEE Trans. Magn. 2014, 50. [CrossRef]

35. Liou, S.H.; Yin, X.; Russek, S.E.; Heindl, R.; Silva, F.; Moreland, J.; Pappas, D.; Yuan, D.P.; Shen, J. Picotesla magnetic sensors for low-frequency applications. IEEE Trans. Magn. 2011, 47. [CrossRef]

36. Guerrero, R.; Pannetier-Lecoeur, M.; Fermon, C.; Cardoso, S.; Ferreira, R.; Freitas, P.P. Low frequency noise in arrays of magnetic tunnel junctions connected in series and parallel. J. Appl. Phys. 2009, 105, 113922. [CrossRef]

37. Leitao, D.C.; Gameiro, L.; Silva, A.V.; Cardoso, S.; Freitas, P.P. Field detection in spin valve sensors using $\mathrm{CoFeB} / \mathrm{Ru}$ synthetic-antiferromagnetic multilayers as magnetic flux concentrators. IEEE Trans. Magn. 2012, 48. [CrossRef]

38. Almeida, J.M.; Freitas, P.P. Field detection in $\mathrm{MgO}$ magnetic tunnel junctions with superparamagnetic free layer and magnetic flux concentrators. J. Appl. Phys. 2009, 105. [CrossRef]

39. Marinho, Z.; Cardoso, S.; Chaves, R.; Ferreira, R.; Melo, L.V.; Freitas, P.P. Three dimensional magnetic flux concentrators with improved efficiency for magnetoresistive sensors. J. Appl. Phys. 2011, 109, $07 \mathrm{E} 521$. [CrossRef]

40. Edelstein, A.; Fisher, G.A. Minimizing $1 / f$ noise in magnetic sensors using a microelectromechanical system flux concentrator. J. Appl. Phys. 2002, 91, 7795-7797. [CrossRef]

41. Edelstein, A.; Fisher, G.A.; Pedersen, M.; Nowak, E.R.; Cheng, S.F.; Nordman, C.A. Progress toward a thousandfold reduction in $1 / f$ noise in magnetic sensors using an AC microelectromechanical system flux concentrator. J. Appl. Phys. 2006, 99, 08B317. [CrossRef]

42. Khoshnoud, F.; de Silva, C.W. Recent advances in MEMS sensor technology-mechanical applications. IEEE Instrum. Meas. Mag. 2012, 15, 14-24. [CrossRef]

43. Ikeda, K.; Kuwayama, H.; Kobayashi, T.; Watanabe, T.; Nishikawa, T.; Yoshida, T.; Harada, K. Three-dimensional micromachining of silicon pressure sensor integrating resonant strain gauge on diaphragm. Sens. Actuators A Phys. 1990, 23, 1007-1010. [CrossRef]

44. Greenwood, J.; Wray, T. High accuracy pressure measurement with a silicon resonant sensor. Sens. Actuators A Phys. 1993, 37-38, 82-85. [CrossRef]

45. Seshia, A.A.; Roessig, T.A.; Howe, R.T.; Gooch, R.W.; Schimert, T.R.; Montague, S. A vacuum packaged surface micromachined resonant accelerometer. J. Microelectromech. Syst. 2002, 11, 784-793. [CrossRef]

46. Wen, M.; Luo, Z.; Wang, W.; Liu, S. A characterization of the performance of MEMS vibratory gyroscope in different fields. In Proceedings of the 15th International Conference on IEEE Electronic Packaging Technology (ICEPT), Chengdu, China, 12-15 August 2014; pp. 1547-1551.

47. Cleland, A.N.; Roukes, M.L. Fabrication of high frequency nanometer scale mechanical resonators from bulk Si crystals. Appl. Phys. Lett. 1996, 69, 2653-2655. [CrossRef]

48. Li, M.; Rouf, V.T.; Jaramillo, G.; Horsley, D.A. MEMS Lorentz force magnetic sensor based on a balanced torsional resonator. Solid-State Sensors. In Proceedings of the 17th International Conference on Solid-State Sensors, Actuators and Microsystems (TRANSDUCERS \& EUROSENSORS XXVII), Barcelona, Spain, 16-20 June 2013; pp. 66-69.

49. Nguyen, C.T.C.; Katehi, L.P.B.; Rebeiz, G.M. Micromachined devices for wireless communications. Proc. IEEE 1998, 86, 1756-1768. [CrossRef]

50. Joshi, M.; Kale, N.; Lal, R.; Rao, V.R.; Mukherji, S. A novel dry method for surface modification of SU-8 for immobilization of biomolecules in Bio-MEMS. Biosens. Bioelectron. 2007, 22, 2429-2435. [CrossRef] [PubMed]

51. Bao, G.; Suresh, S. Cell and molecular mechanics of biological materials. Nat. Mater. 2003, 2, 715-725. [CrossRef] [PubMed]

52. Gau, J.J.; Lan, E.H.; Dunn, B.; Ho, C.M.; Woo, J.C.S. A MEMS based amperometric detector for E. coli bacteria using self-assembled monolayers. Biosens. Bioelectron. 2001, 16, 745-755. [CrossRef]

53. Gaspar, J.; Li, H.; Freitas, P.P.; Chu, V.; Conde, J.P. Integrated magnetic sensing of electrostatically actuated thin-film microbridges. J. Microelectromech. Syst. 2003, 12, 550-556. [CrossRef]

54. Edelstein, A.S.; Burnette, J.E.; Fischer, G.A.; Olver, K.; Egelhoff, W., Jr.; Nowak, E.; Cheng, S.F. Validation of the microelectromechanical system flux concentrator concept for minimizing the effect of $1 / f$ noise. J. Appl. Phys. 2009, 105, 07E720. [CrossRef]

55. Guedes, A.; Patil, S.B.; Cardoso, S.; Chu, V.; Conde, J.P.; Freitas, P.P. Hybrid magnetoresistive/microelectromechanical devices for static field modulation and sensor $1 / f$ noise cancellation. J. Appl. Phys. 2008, 103, 07E924. [CrossRef] 
56. Guedes, A.; Jaramillo, G.; Buffa, C.; Vigevani, G.; Cardoso, S.; Leitao, D.C.; Freitas, P.P.; Horsley, D.A. Towards picoTesla Magnetic Field Detection Using a GMR-MEMS Hybrid Device. IEEE Trans. Magn. 2012, 48, 4115-4118. [CrossRef]

57. Guedes, A.; Patil, S.; Wisniowski, P.; Chu, V.; Conde, J.P.; Freitas, P.P. Hybrid Magnetic Tunnel Junction-MEMS High Frequency Field Modulator for $1 / f$ Noise Suppression. IEEE Trans. Magn. 2008, 44, 2554-2557. [CrossRef]

58. Hu, J.; Pan, M.; Tian, W.; Chen, D.; Luo, F. Integrating magnetoresistive sensors with microelectromechanical systems for noise reduction. Appl. Phys. Lett. 2012, 101, 234101. [CrossRef]

59. Hu, J.; Pan, M.; Tian, W.; Chen, D.; Zhao, J. Magnetostatic detection using magnetoresistive sensors with vertical flux modulation. Rev. Sci. Instrum. 2012, 83, 055009. [CrossRef] [PubMed]

60. Hu, J.; Pan, M.; Tian, W.; Chen, D.; Zhao, J.; Luo, F. $1 / f$ noise suppression of giant magnetoresistive sensors with vertical motion flux modulation. Appl. Phys. Lett. 2012, 100, 244102. [CrossRef]

61. Patil1, S.; Guedes, A.; Freitas, P.P.; Cardoso, S.; Chu, V.; Conde, J.P. On-chip magnetoresistive detection of resonance in microcantilevers. Appl. Phys. Lett. 2009, 95, 023502. [CrossRef]

62. Jander, A.; Nordman, C.A.; Pohm, A.V.; Anderson, J.M. Chopping techniques for low-frequency nanotesla spin-dependent tunneling field sensors. Appl. Phys. Lett. 2003, 93, 8382-8384. [CrossRef]

63. Kobayashi, N.; Ohnuma, S.; Murakami, S.; Masumoto, T.; Mitani, S.; Fujimori, H. Enhancement of low-field-magnetoresistive response of tunnel-type magnetoresistance in metal-nonmetal granular thin films. J. Magn. Magn. Mater. 1998, 188, 30-34. [CrossRef]

64. Freitas, P.P.; Costa, J.L.; Almeida, N.; Melo, L.V.; Silva, F.; Bernardo, J.; Santos, C. Giant magnetoresistive sensors for rotational speed control. J. Appl. Phys. 1999, 85, 5459-5461. [CrossRef]

65. Leitao, D.C.; Coelho, P.; Borme, J.; Knudde, S.; Cardoso, S.; Freitas, P.P. Ultra-Compact $100 \times 100 \mu \mathrm{m}^{2}$ Footprint Hybrid Device with Spin-Valve Nanosensors. Sensors 2015, 15, 30311-30318. [CrossRef] [PubMed]

66. Haykin, S.; Moher, M. Introduction to Analog \& Digital Communications, 2nd ed.; John Wiley \& Sons: Hoboken, NJ, USA, 2012.

67. Lathi, B.P. Modern Digital and Analog Communication Systems, 3rd ed.; Oxford University Press: Oxford, UK, 1998.

68. Ozdemir, S.; Akhtar, S.; Gunal, O.E.; Khater, M.E.; Saritas, R.; Abdel-Rahman, E.M.; Yavuz, M. Measuring the Quality Factor in MEMS Devices. Micromachines 2015, 6, 1935-1945. [CrossRef]

69. Le Foulgoc, B.; Bourouina, T.; Le Traon, O.; Bosseboeuf, A.; Marty, F.; Breluzeau, C.; Grandchamp, J.P.; Masson, S. Highly decoupled single-crystal silicon resonators: An approach for the intrinsic quality factor. J. Micromech. Microeng. 2006, 16, S45-S53. [CrossRef]

70. Cheng, S.F.; Lubitz, P.; Zheng, Y.; Edelstein, A.S. Effects of spacer layer on growth, stress and magnetic properties of sputtered permalloy film. J. Magn. Magn. Mater. 2004, 282, 109-114. [CrossRef]

71. Klaasse, G.; Puers, R.; Tilmans, H.A.C. Piezoelectric versus electrostatic actuation for a capacitive RF-MEMS switch. In Proceedings of the 3rd Workshop on Semiconductor Sensor and Actuator, Leuven, Belgium, 29 November 2002; pp. 631-634.

72. Alves, F.S.; Dias, R.A.; Cabral, J.; Gaspar, J.; Rocha, L.A. High-resolution MEMS inclinometer based on pull-in voltage. IEEE J. Microlectromech. Syst. 2105, 24, 931-939. [CrossRef]

73. Gaspar, J.; Chu, V.; Conde, J.P. Amorphous silicon electrostatic microresonators with high quality factors. Appl. Phys. Lett. 2004, 84, 622-624. [CrossRef]

74. Pedrini, G.; Gaspar, J.; Tu, W.; Osten, W.; Paul, O. Calibration of optical systems for the measurement of microcomponents. Opt. Lasers Eng. 2009, 47, 203-210. [CrossRef]

75. Bartsch, U.; Gaspar, J.; Paul, O. Low-frequency two-dimensional resonators for vibrational micro energy harvesting. J. Micromech. Microeng. 2010, 20, 035016. [CrossRef]

76. Passi, V.; Sodervall, U.; Nilsson, B.; Petersson, G.; Hagberg, M.; Krzeminski, C.; Dubois, E.; Du Bois, B.; Raskin, J.-P. Anisotropic vapor HF etching of silicon dioxide for Si microstructure release. Microelectron. Eng. 2012, 95, 83-89. [CrossRef]

77. Hooda, M.K.; Wadhwa, M.; Verma, S.; Nayak, M.M.; George, P.J.; Paul, A.K. A systematic study of DRIE process for high aspect ratio microstructuring. Vacuum 2010, 84, 1142-1148. [CrossRef]

(C) 2016 by the authors; licensee MDPI, Basel, Switzerland. This article is an open access article distributed under the terms and conditions of the Creative Commons Attribution (CC-BY) license (http:/ / creativecommons.org/licenses/by/4.0/). 